\title{
Atomistic Dislocation Core Energies and Calibration of Non-singular Discrete Dislocation Dynamics
}

\author{
Yi Hu${ }^{1}$, B. Szajewski ${ }^{2}$, D. Rodney ${ }^{3}$, W. A. Curtin ${ }^{1}$ \\ ${ }^{1}$ Laboratory for Multiscale Mechanics Modeling (LAMMM) and National Centre for \\ Computational Design and Discovery of Novel Materials (NCCR MARVEL), École \\ Polytechnique Fédérale de Lausanne, 1015 Lausanne, Switzerland \\ ${ }^{2}$ Simulation Sciences Branch, U.S. Army Research Laboratory, Aberdeen Proving \\ Ground, MD, 21005-5066, USA \\ ${ }^{3}$ Institut Lumière, Matière, UMR 5306 Universitè de Lyon, F-69622 Villeurbanne, \\ France \\ E-mail: yi.hu@epfl.ch
}

\begin{abstract}
The total energy of an atomistic dislocation includes contributions from the inelastic/large-distortion "core" region. Capturing this inelastic "core" energy is important, especially for dislocations with a curvature in the 10-100 nm scale. Current implementations of discrete dislocation dynamics (DDD) mesoscale simulations either approximate or neglect the core energy and so do not provide consistency with fullyatomistic studies. Using established interatomic potentials for FCC metals, the total dislocation energy is computed directly in atomistic simulations of straight dislocations and a core energy at any desired cut-off core radius is obtained as a function of dislocation character. A proper introduction of the atomistic core energy into the ParaDiS DDD code that uses a non-singular theory [1] is then presented. The resulting atomistically-informed ParaDiS DDD is used to simulate the periodic bow-out of edge and screw dislocations in near-elastically-isotropic Aluminum at various length and stress, with comparisons to fully-atomistic simulations. Generally good agreement is obtained between DDD and atomistics, with the best agreement achieved using a nonsingular regularization parameter in the range of $a=5-10 b$. The analysis is then extended to compute the core energy of the Shockley partial dislocations that arise in the dissociation of perfect dislocations in fcc metals.
\end{abstract}

Keywords: dislocation line tension, discrete dislocation dynamics, atomistic simulation

Submitted to: Modelling Simul. Mater. Sci. Eng.

\section{Introduction}

Dislocations are the line defects that carry the plastic displacement in plasticallydeforming crystalline materials. The dislocation line is the line of termination of crystal slip across a glide plane, and this termination generates a singularity and long-range 
elastic fields. The singularity is resolved at the atomic scale as determined by atomistic interactions. The total energy of a dislocation can be conceptually partitioned into two parts [2]: an elastic energy contribution due to the elastic deformations introduced by the dislocation and an inelastic energy contribution occuring in the dislocation core region, loosely defined as the region where the non-linear/non-convex energy landscape of the material and discreteness of the lattice structure influence the dislocation structure and fields. The elastic energy dominates the total energy of an infinite straight dislocation, diverging in an infinite medium, often leading to approximations that neglect the inelastic core energy. However, when the dislocation density is high, dislocation segments are short and close to one another, and/or when the dislocation radius of curvature is in the sub-micron scale of $100 \mathrm{~nm}$ and below, the atomistic core energy contribution cannot be neglected a priori.

Core effects at the scale of the dislocation Burgers vector $b$ are automatically included in fully-atomistic studies. The scales accessible in such simulations are practically limited to below $10^{7}$ atoms, and hence lengths of only a few hundred atomic spacings. Such scales are often too small to capture the full complexity of dislocation network evolution typical of real materials. For this reason, mesoscale Discrete Dislocation Dynamics (DDD) $[3,4,5,6,7]$ have emerged to model the collective behavior of dislocations up to scales of 10-50 microns [8]. In most mesoscale models, dislocation lines are discretized into finite segments and the Peach-Koehler driving forces on the segments are computed from the elastic interactions among all segments in the system. The segments are treated using continuum fields, appropriate for the longrange elastic interactions, and the singularity at the origin is circumvented by using a heuristic cutoff radius or a regularized field theory $[1,9]$. Details of the actual dislocation core structure and energy are usually neglected, although occasionally the dissociated structure of glissile fcc dislocations is incorporated by treating each partial dislocation as a separate dislocation line [10]. A core energy may also be introduced [3], but is treated as a parameter, while a regularized core model automatically imposes some core energy that is not necessarily directly related to any atomistic quantity. Multiscale methods, which aim to combine both atomistic and continuum descriptions simultaneously in different regions of the material [11, 12] also require a careful calibration of the continuum description to the atomistic dislocations, including the core energy. Accurate computation of the core energy and precise calibration of the non-singular continuum theory are the topics of this paper.

There are two basic approaches to computing the dislocation core energy. One method uses a single straight dislocation in a cylindrical geometry. This method is mentioned in [2] but has not been widely used. We use this method here because (i) it is easy to implement, (ii) requires no additional corrections, (iii) has a controllable accuracy and easy convergence assessment because the scaling of the far-field elastic energy versus distance is known analytically, and (iv) it is easy to calibrate non-singular theory to the atomistic results. The other method uses a dislocation dipole geometry [40]. The dipole method has been preferred by many researchers when using first 
principles methods [41, 15] because the periodicity enables accurate computation of the total system energy. The dipole method has also been employed in molecular statics studies $[14,18]$. The dipole method requires corrections for the periodic images, which can be computed numerically in anisotropic elasticity. This method is thus accurate if the dislocations interact solely through the elastic fields, which must be verified by convergence studies. Precise calibration of non-singular theory is less direct and has not, to our knowledge, been done to date.

There have also been significant efforts to describe the dislocation core region itself through approximate (non-atomistic) models, e.g. [43, 39, 42, 19]. These elegant methods help bridge the gap between atomistic dislocations and their continuum counterparts, but do not usually ensure that the total dislocation energy is preserved in making such a transition. Thus, the specific determination of the core energy and its precise introduction into a higher-scale method has not generally been achieved, with the exception of the recent work of Geslin et al. [19]. Therefore, in this paper, we execute a straightforward strategy for computing the dislocation core energy in a full atomistic environment, discuss the calibration of non-singular theory to atomistics, and demonstrate that the atomistically-calibrated non-singular theory can provide good predictions of the evolving curvature of a dislocation over scales of $\sim 25-50 \mathrm{~nm}$. This work largely resolves previous discrepancies between the atomistics and DDD studies using an approximate core-energy representation [20] and demonstrates a general methodology that can be applied to any system described by interatomic potentials.

The remainder of this paper is organized as follows. In the next section, we introduce the basics of dislocation energies and the atomistic simulation method, and compute the dislocation core energies for $\mathbf{b}=a[\overline{1} 10] / 2$ dislocations in the fcc metals $\mathrm{Al}, \mathrm{Cu}$, and $\mathrm{Ni}$. In section 3 , we present the resulting atomistic core contributions to the dislocation line tension. In section 4, we discuss how to incorporate the atomistic core energy into the non-singular theory of dislocations [1]. In section 5, we define the bow-out dislocation test problem and compare predictions of DDD using non-singular theory [3] and atomistics, drawing attention to the role of the regularization parameter in the non-singular theory. In section 6 , we compute the atomistic core energies for the $\mathbf{b}_{\mathrm{s}}=a[\overline{2} 11] / 6$ Shockley partial dislocations in fcc materials as appropriate for use in continuum-level models. In section 7 , we discuss our results and restate our major conclusions.

\section{Atomistic calculation of dislocation core energies}

For an infinite straight dislocation, the total dislocation energy within a cylinder of radius $R$ centered on the dislocation line can be expressed as

$$
E(\theta)=K(\theta) \ln \left(\frac{R}{r_{\mathrm{c}}}\right)+\left.E_{\mathrm{c}}(\theta)\right|_{r_{\mathrm{c}}}
$$

where $\theta$ is the character angle of the dislocation and $E_{\mathrm{c}}$ is the core energy defined at a chosen cutoff radius $r_{c}$. The separation between elastic and core energies is 
always defined with respect to some chosen cutoff; for straight dislocations, there is no strict nor unique partition of the total energy into these two components. The goal of calibrating the core energy is to capture the correct total dislocation energy at large $R \gg b$, independent of the chosen cutoff $r_{\mathrm{c}}$. The coefficient $K(\theta)$ is fully determined by the anisotropic elastic constants, dislocation Burgers vector $\mathbf{b}$, and dislocation line direction can be computed for any particular material using the Stroh formalism [21]. Note that the dislocation is treated as a full undissociated dislocation, and thus the core energy as defined here includes, for fcc dislocations, the energies of the two partial dislocations, their interactions, and the stacking fault energy, as well as all non-linear contributions. Inclusion of all of these effects into the core energy is consistent with most DDD simulations that treat the dislocation as a single undissociated dislocation; the core energies of the partial dislocations in fcc metals are presented in section 6 .

We compute the core energy $E_{\mathrm{c}}(\theta)$ atomistically by first measuring the total dislocation energy as a function of $R$ in an atomistic simulation. We then extrapolate the far-field elastic energy back to the chosen cutoff radius $r_{\mathrm{c}}$. Here, we choose $r_{\mathrm{c}}=b$. The core energy at any other chosen cutoff $r_{\mathrm{c}}$ can then be obtained with no further approximation by writing the total energy as

$$
\begin{aligned}
E(\theta) & =K(\theta) \ln \left(\frac{R}{b}\right)+\left.E_{\mathrm{c}}(\theta)\right|_{b} \\
& =K(\theta) \ln \left(\frac{R}{r_{\mathrm{c}}}\right)+K(\theta) \ln \left(\frac{r_{\mathrm{c}}}{b}\right)+\left.E_{\mathrm{c}}(\theta)\right|_{b} \\
& =K(\theta) \ln \left(\frac{R}{r_{\mathrm{c}}}\right)+\left.E_{\mathrm{c}}(\theta)\right|_{r_{\mathrm{c}}}
\end{aligned}
$$

That is, a term $K(\theta) \ln \left(r_{\mathrm{c}} / b\right)$ is subtracted from the elastic energy and added to the core energy; the total energy remains unchanged.

A schematic of the simulation setup is shown in figure 1. The atomistic simulation model is a cylindrical disk of outer radius $R_{\max }$ aligned along the dislocation line direction. Periodic boundary conditions are imposed along the dislocation line direction. Fixed displacement boundary conditions are applied to atoms on an outer annulus of the cylindrical disk. Specifically, to the cylinder of radius $R_{\max }$ we add an annulus of atoms of thickness $d_{\text {bd }}$ equal to twice the cutoff distance of the chosen interatomic potential. The position of the atoms in this annular domain are held fixed at the positions predicted by the anisotropic elastic solution for a Volterra dislocation at the origin as obtained using Stroh's formalism. The outer boundary condition is thus exactly the field associated with a singular core; differences due to core dissocation are dipolar in nature and so do not affect the boundary conditions if $R_{\max }$ is large enough. All atoms within $R_{\max }$ are then relaxed fully to their zero-force positions, yielding the minimum-energy structure for the dislocation.

The details of the atomistic calculations are as follows. We measure the total atomic energy of those atoms within radius $R$ as a function of $R$ measured from the geometrical center of the dislocation core. We compute the energy at 50 different values of $R$ from $b$ to, typically, $R_{\max }=450 b$ with equal spacing in $\ln (R / b)$. At each $R$, there are $N$ atoms 
within $R$ and we subtract the reference total energy $N E_{0}$ where $E_{0}$ is the energy per atom of an unstrained fcc crystal (the cohesive energy). The resulting total dislocation energy in the region $r \leqslant R$ is then obtained. In figure 1 an example demonstrating the energy versus $\ln (R / b)$ for screw and edge dislocations in $\mathrm{Ni}$ obtained using the Mishin potential [16] is shown.

According to elasticity theory (see (1)), for $R$ much larger than the core region, the total energy versus $\ln (R / b)$ should be linear with a slope of precisely $K(\theta)$. The measured slopes computed from the simulation results at radius $R$, using the last 10 points including $r=R$, are shown in table 1 for various $R$. For $R=100 b$, the error in $K(\theta)$ is $\approx 1 \%$, decreasing to $\approx 0.1 \%$ at $R=R_{\max }=500 b$. While these errors seem small, it is crucial to recognize that the core energy is obtained by extrapolating back to $R=b$, and the error in extracting the core energy is thus greatly magnified. Table 1 shows the extrapolated core energy using the $K(\theta)$ estimated from the simulations at various $R$. The core energy converges with increasing $R$. Setting the core energy at $R=500 b$ as a standard core energy, we can compute the error for core energies computed at smaller $R$. For the edge dislocation the error in the core energy at $R=100 b$ is $\approx 10 \%$. The error reduces to less than $1 \%$ at $R=400 \mathrm{~b}$. Thus, it is necessary to use radii of $450 \mathrm{~b}$ or larger to make accurate core energy estimates. Fortunately, atomistic computations at these sizes are not computationally challenging when using EAM and MEAM potentials.

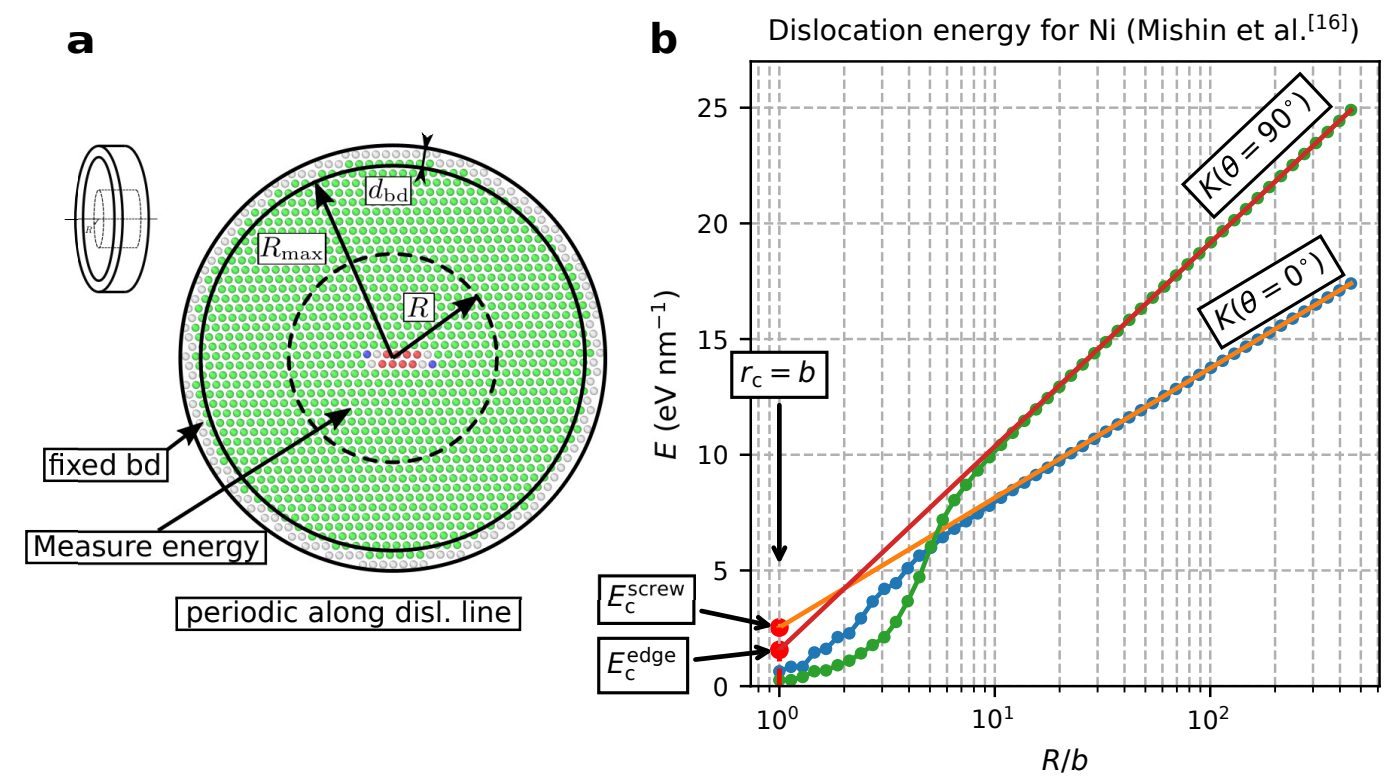

Figure 1. (a) Schematic of the simulation model with simulation size $R_{\max }+d_{\mathrm{bd}}$, outer boundary layer thickness $d_{\mathrm{bd}}$, and radius $R$ for measuring energy, as indicated. This is an artificially small $R_{\max }$ used only to show actual atoms, which are colored using common neighbor analysis so that the dissociated core of the fcc edge dislocation is evident at this scale. (b) Total energy within radius $r \leqslant R$ for screw and edge dislocations computed using the Mishin Ni EAM potential [16]. The dislocation core energy at cutoff $r_{\mathrm{c}}=b$ is obtained by extrapolating the elastic response at large $R$ back to $R=b$, as indicated. 
Table 1. Elasticity coefficient $K(\theta)$ and estimated dislocation core energy as a function of radius $R$, for edge and screw dislocations using the Mishin Ni EAM potential (Mishin et al. [16]. At each $R$, the $K(\theta)$ is computed by measuring slope of the the last 10 points in the energy plot and is compared to the exact value predicted by anisotropic elasticity. The estimated core energy evaluated at cutoff $r_{\mathrm{c}}=b$ is shown for each $R$, showing the convergence of the core energy to a reliable value at the largest $R$ values.

\begin{tabular}{lllllll}
\hline \multirow{2}{*}{$R_{\max }(\mathrm{b})$} & \begin{tabular}{l}
$K(\theta) /\left(b^{2} / 4 \pi\right)$ \\
\cline { 2 - 7 }
\end{tabular} & $K(\theta)$ error & $\begin{array}{l}E_{\mathrm{c}}(\theta) \\
(\mathrm{eV} / \mathrm{nm})\end{array}$ & $\begin{array}{l}K(\theta) /\left(b^{2} / 4 \pi\right) \\
(\mathrm{GPa})\end{array}$ & $K(\theta)$ error & $\begin{array}{l}E_{\mathrm{c}}(\theta) \\
(\mathrm{eV} / \mathrm{nm})\end{array}$ \\
\hline 100 & 124.966 & $0.88 \%$ & 1.193 & 79.827 & $1.02 \%$ & 1.797 \\
\hline 200 & 124.464 & $0.48 \%$ & 1.254 & 79.326 & $0.39 \%$ & 1.859 \\
\hline 300 & 124.275 & $0.33 \%$ & 1.282 & 79.181 & $0.20 \%$ & 1.880 \\
\hline 400 & 124.180 & $0.25 \%$ & 1.297 & 79.113 & $0.12 \%$ & 1.891 \\
\hline 500 & 124.086 & $0.17 \%$ & 1.313 & 79.069 & $0.06 \%$ & 1.899 \\
\hline elasticity & 123.870 & - & - & 79.019 & - & - \\
\hline
\end{tabular}

Extending the analysis across the full range of dislocation character angles, we use a simulation system size $R_{\max }=450 b$ to achieve sufficient accuracy for $E_{\mathrm{c}}(\theta)$. For each character angle, the appropriate periodic distance along the dislocation line must be determined from crystallography and used, increasing the size of the atomistic system. Nonetheless, the maximum number of atoms in any simulation in this paper is 15 million atoms for character angle at $47.8^{\circ}$, still computationally accessible. The computed total energy versus $\ln (R / b)$ for all character angles studied here is shown for Ni in figure 2 .

Extrapolation using the slope $K(\theta)$ yields the associated core energy for each character angle as shown in figure 3 for the Mishin $\mathrm{Cu}, \mathrm{Ni}$, and $\mathrm{Al}$ potentials [16] and the Ercolessi-Adams Al potential [17]. The general trends are similar across the different metals. The behavior observed for the Mishin Al potential is notably different from those of the other three potentials, however. This suggests that this potential should be used with caution for the intermediate character angles and especially the commonly-studied $30^{\circ}$ case. For $\mathrm{Cu}$, the screw core energy at $r_{\mathrm{c}}=b$ is negative but this has no physical importance as it is simply the correction needed, as a core energy, to ensure that the total dislocation energy is identical to the atomistic result. Selecting a larger cutoff $r_{\mathrm{c}}>b$ would render the core energy positive (see (2)) with no change in total energy or physics of the problem. The magnitudes of the core energies differ, but the screw core energies at $r_{\mathrm{c}}=b$ scale roughly with the shear moduli of the different metals. In the DDD code ParaDiS, the default core energy is $\mu b^{2} / 4 \pi \ln \left(r_{\mathrm{c}} / 0.1 b\right)\left(\cos ^{2} \theta+\sin ^{2} \theta /(1-\nu)\right)$ [22]. Table 2 shows the atomistic and ParaDis core energies for screw and edge dislocations at $r_{\mathrm{c}}=b$. The differences are significant, especially for the edge dislocations. The nonsingular theory in ParaDis has additional core energy contributions as discussed in detail below, so the comparison in table 2 is not a precise quantitative comparison but the significant differences strongly motivate improved core models for ParaDis calibrated 
to atomistics, which is a main focus of this paper.

Table 2. Comparison of the atomistic core energy with the default core energy in ParaDis.

\begin{tabular}{lcccc}
\hline \multirow{2}{*}{$(\mathrm{eV} / \mathrm{nm})$} & \multicolumn{2}{c}{$\theta=0^{\circ}$} & \multicolumn{2}{c}{$\theta=90^{\circ}$} \\
\cline { 2 - 5 } & atomistic & ParaDiS & atomistic & ParaDiS \\
\hline $\mathrm{Cu}$ Mishin[16] & 3.088 & 1.071 & -0.044 & 5.314 \\
\hline Ni Mishin[16] & 5.312 & 2.535 & 1.566 & 8.472 \\
\hline Al Ercolessi-Adams[17] & 2.862 & 0.810 & 0.463 & 4.370 \\
\hline Al Mishin[16] & 2.609 & 1.262 & 1.198 & 4.020 \\
\hline
\end{tabular}

For a compact representation of the core energies, figure 3 also shows numerical fits to the atomistic core energies at $r_{\mathrm{c}}=b$ using a form consistent with the symmetries of the problem [18] as

$$
E_{\mathrm{c}}^{\mathrm{fit}}(\theta)=a_{n}\left(\cos ^{2} \theta\right)^{n}+a_{n-1}\left(\cos ^{2} \theta\right)^{n-1}+\ldots+a_{0}
$$

The non-zero coefficients in the fit for each material are indicated in each figure. The simple decomposition of the core energy into screw and edge components as $E_{\mathrm{c}}^{\mathrm{fit}}(\theta)=E_{\mathrm{s}} \cos ^{2} \theta+E_{\mathrm{e}} \sin ^{2} \theta$ might be valid for some potentials, e.g. the $\mathrm{Cu}$ Mishin potential, but the more precise functional form of (3) easily obtained and is generally needed to capture the curvature of core energy, which contributes to the line tension (see section 3). 


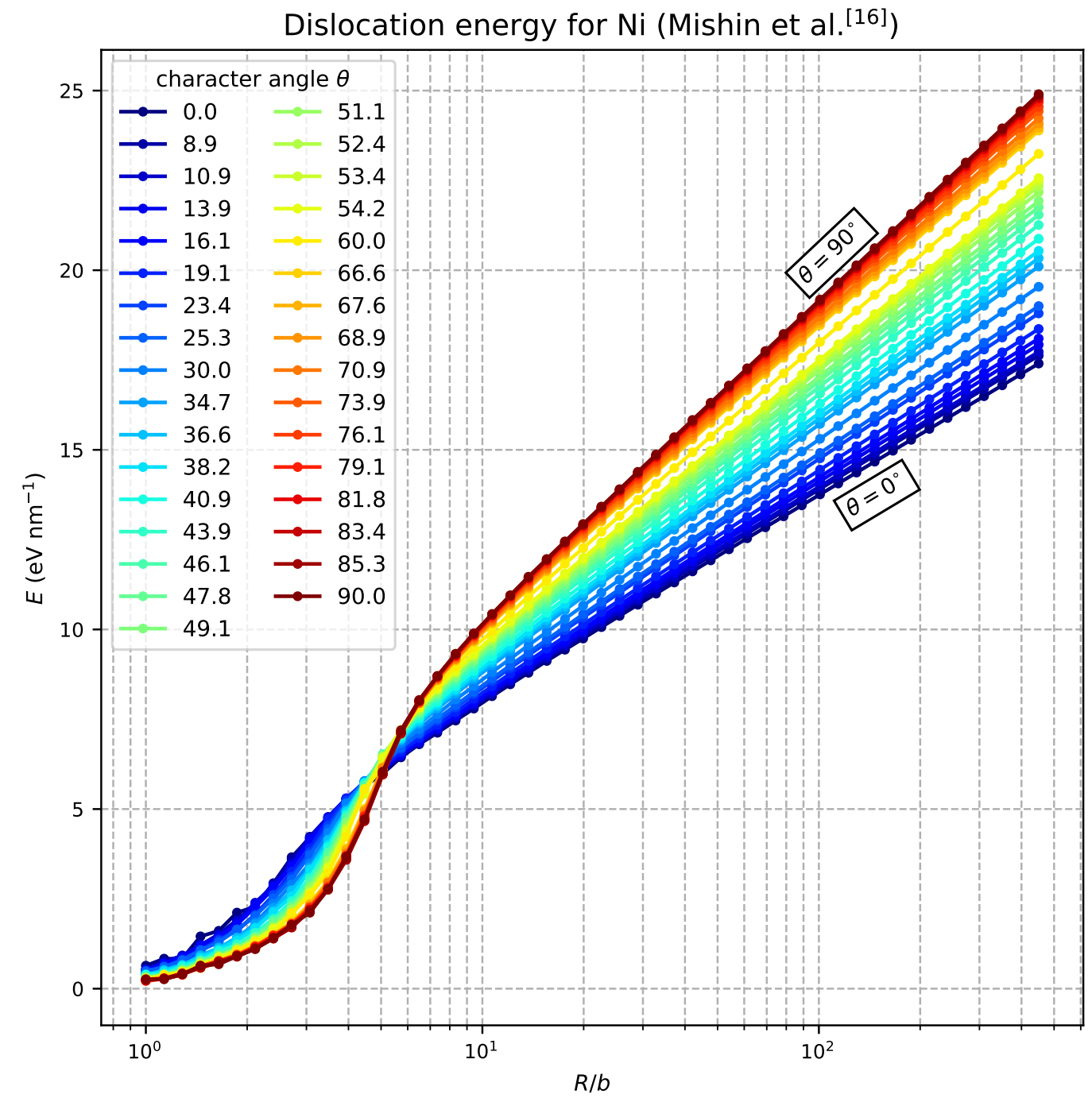

Figure 2. Total dislocation energy versus radius $R \leq 450 b$ for 33 different dislocation character angles (units in degrees), using the Mishin EAM Ni potential (Mishin et al. [16]) 

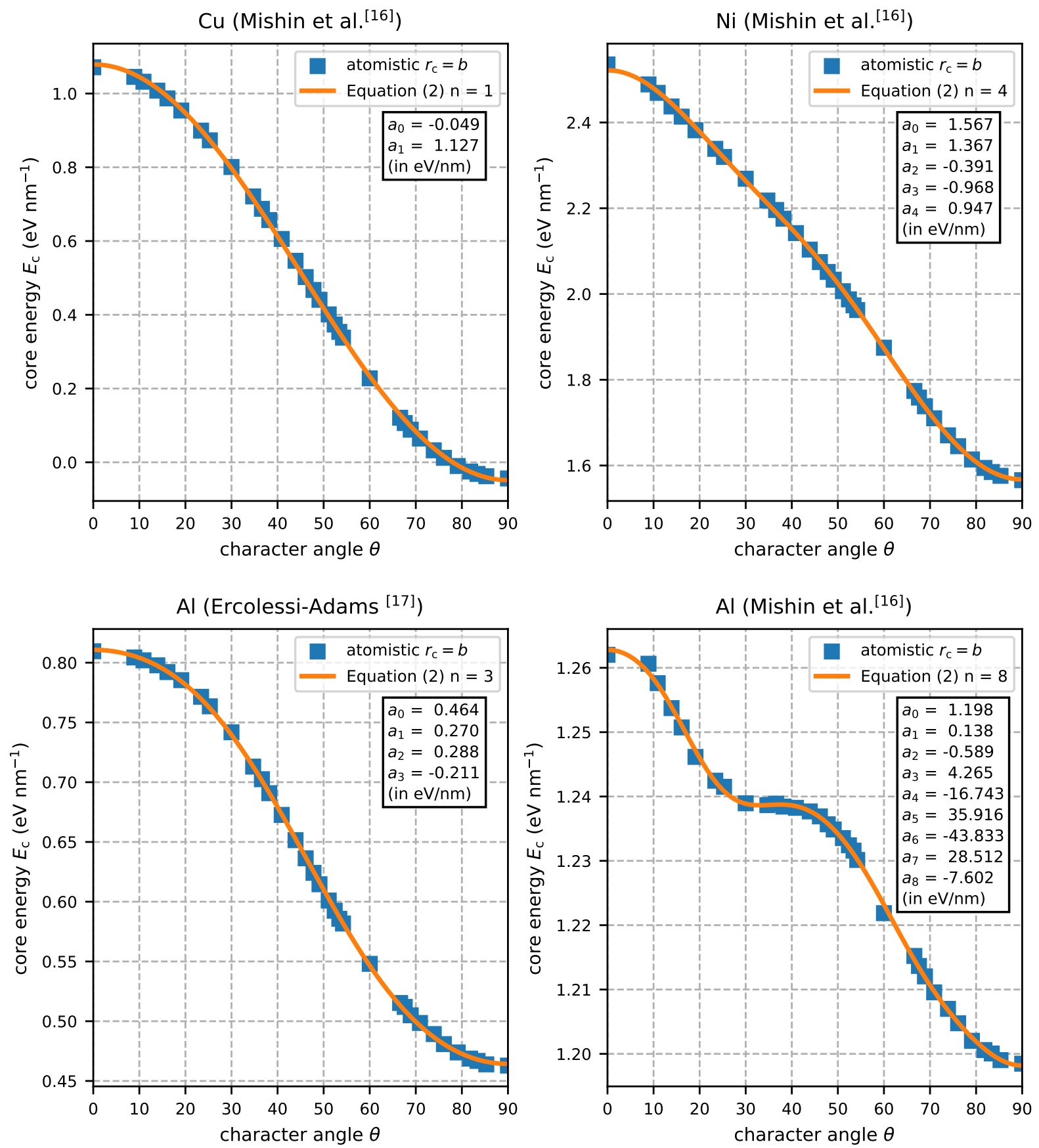

Figure 3. Atomistic core energy for the full dislocation at cutoff $r_{\mathrm{c}}=b$ for $\mathrm{Cu}, \mathrm{Ni}$, and Al metals as a function of character angle. The solid line shows a fit to an approximate form (3) with coefficients $a_{i}, i=0 \ldots n$ as indicated.

\section{Atomistic core contributions to dislocation line tension}

The behavior of curved dislocations is often discussed using the concept of line tension. The line tension of a dislocation is often interpreted simply as a force per unit line length of dislocation due to the change of dislocation geometry. Such an approximate 
concept is very useful for baseline analytic models of moderately complex dislocation geometries, capturing the general scaling of behavior versus total dislocation length. Strictly, the line tension is a configurational force for a given dislocation geometry. As such, the line tension is defined as the change of dislocation energy with respect to the change in dislocation length due to an infinitesimal change in the dislocation geometry while maintaining an overall parametric configuration of the dislocation. Denoting the dislocation geometry by the configuration label $X$, the line tension $\Gamma_{X}$ is expressed in terms of the change in total dislocation energy $W$ divided by the change of total dislocation length $L$,

$$
\Gamma_{X}=\frac{\delta W}{\delta L}
$$

A proper line tension is thus defined for each specific type of dislocation geometry $X$ (e.g. circular loop, small triangular bow-out, small sinusoidal bow-out) [23]. If a dislocation geometry is composed of different parts, the change in total dislocation energy $W$ involves changes in the self energy of each part plus the elastic interactions among the different parts. In previous work, the line tension was evaluated in the context of elasticity theory accounting only for the change in total elastic dislocation energy. The change in dislocation core energy is often neglected. However, an accurate line tension requires the contribution due to the core energy. This was recently confirmed [19] by analyzing the thermal fluctuations of dislocations in MD simulations. This work showed that the fluctuation spectra can be explained using linear elasticity for the long-range interactions supplemented by a core line tension to account for short-range core effects.

If the interaction energy is neglected, the line tension of a geometry can be expressed using the self energy of a dislocation per unit length $E$ as

$$
\Gamma_{\text {self }}=E+\frac{\partial^{2} E}{\partial \theta^{2}}
$$

A standard derivation can be found in [2], and a more general derivation is given in [24]. Since the dislocation core energy $E_{\mathrm{c}}$ is one component of the dislocation self energy, the core energy contribution to the dislocation line tension is

$$
\Gamma_{\mathrm{c}}=E_{\mathrm{c}}+\frac{\partial^{2} E_{\mathrm{c}}}{\partial \theta^{2}}
$$

The core energy contributions to the line tension for the materials studied here are shown in figure 4 . We see that the line tension contribution from the core energy can vary substantially in magnitude as the dislocation character is varied, and can be a rather complex function of the character angle. Importantly, even when the core energy might be reasonably described by the decomposition $E_{\mathrm{c}}^{\mathrm{fit}}(\theta)=E_{\mathrm{s}} \cos ^{2} \theta+E_{\mathrm{e}} \sin ^{2} \theta$ (e.g. Ni, Al), the line tension contribution from core energy can have a more complex dependence on character angle due to the inclusion of the curvature of the core energy expressed in (6). These results pertain to the chosen cutoff $r_{c}=b$. Increasing the cutoff distance embeds more "elastic" energy into the "core energy". These elastic contributions scale with $K(\theta) \ln \left(r_{c} / b\right)$ (see (2)) and so make the core contribution increasingly follow the 
trend of the elastic term with $K(\theta)$. However, the underlying dependence of the total line tension on the core contribution computed at $r_{c}=b$ (figure 4 ) does not disappear.
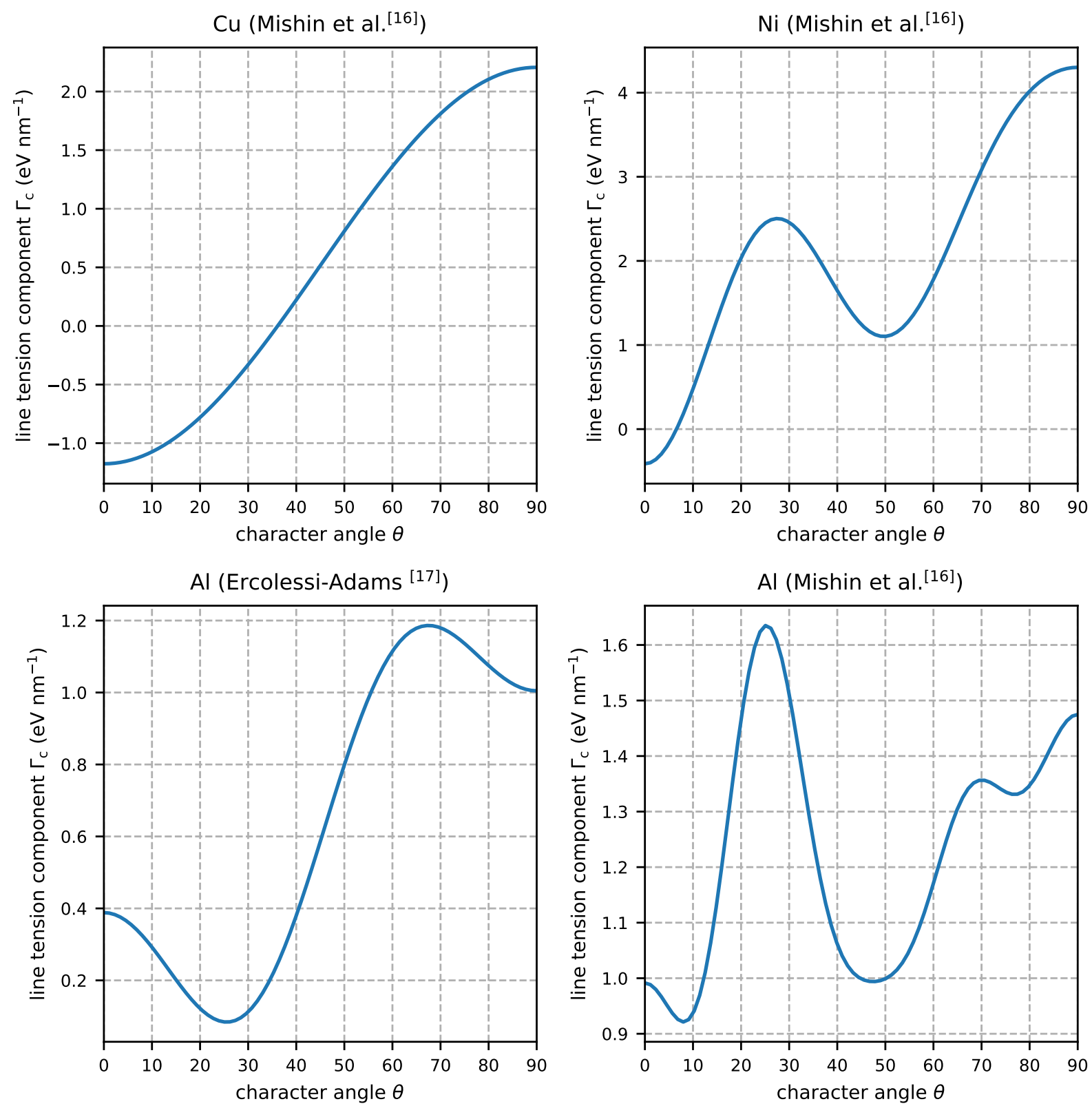

Figure 4. Line tension calculation using (6) and the core energy approximation in (3) with the fitted parameters given in figure 3 . The cutoff radius is $r_{\mathrm{c}}=b$.

To examine the relative importance of the core and elasticity contributions to the line tension at $r_{c}=b$, we consider the periodic sinusoidal bow-out geometry. This geometry was studied by Lothe [25] for a Volterra dislocation in an isotropic elastic material; a more general derivation is presented in the supplement of [19] within a 
non-singular theory. The elastic contribution to the line tension is

$\Gamma_{\text {elas }}^{\text {sine }}=\frac{\mu b^{2}}{4 \pi(1-\nu)}\left[2\left(1-\nu \sin ^{2} \theta\right) \ln \left(\frac{L}{2 \pi \mathrm{e}^{\gamma_{0}} \rho}\right)-\left(1-\nu \cos ^{2} \theta\right) \ln \left(\frac{L}{2 \pi \mathrm{e}^{\gamma_{0}-1 / 2} \rho}\right)\right]$

where $\gamma_{0}$ is the Euler-Mascheroni constant and $\mathrm{e}^{\gamma_{0}} \approx 1.78$. To relate eq. (7) to our atomistic analysis, the interaction cutoff $\rho$ must be set to $\rho=\frac{1}{2} r_{\mathrm{c}}[2]$. To make a quantitative comparison, we consider the Al Mishin potential [16] with isotropic shear modulus $\mu_{\mathrm{s}}=28.7 \mathrm{GPa}$ and Poisson's ratio $\nu_{\mathrm{s}}=0.348$ (see section 5) and typical dislocation densities of deformed metals of $10^{12} \sim 10^{15} \mathrm{~m}^{-2}$ corresponding to dislocation lengths between forest intersections of $30 \sim 1000 \mathrm{~nm}$. For the edge dislocation, the relative contribution of the core energy to the elastic energy, $\Gamma_{\mathrm{c}} / \Gamma_{\text {elas }}^{\text {sine }}$, is 2.11 at 30 $\mathrm{nm}$ and 0.57 at $1000 \mathrm{~nm}$. Thus the core contribution for the edge is significant over the entire range of metallurgically-relevant lengths and strengthening processes (see, for instance, [26]). For the screw dislocation, the core contributions are 0.15 at $30 \mathrm{~nm}$ and 0.07 at $1000 \mathrm{~nm}$, and so are less important in general. Thus, if accurate predictions for metallurgical plasticity phenomena are to be made using dislocation-based analyses, the contribution of the core energy is essential.

\section{Calibrating non-singular theory to atomistic core energies}

With the importance of the core energy established, we now address how to calibrate the non-singular dislocation theory of Cai et al. [1] that is implemented in the DDD code ParaDis to the "true" atomistic result.

A non-singular istotropic theory was proposed by Cai et al. [1] to eliminate the energy and stress divergences at the singular core of the Volterra dislocation. This was accomplished by assuming that the dislocation Burgers vector is spatially distributed over all space according to a radial distribution function $\tilde{w}(r ; a)$ satisfying

$$
\mathbf{b}=\int \mathbf{b} \tilde{w}(r ; a) \mathrm{d}^{3} \mathbf{x}
$$

where $a$ is introduced as the regularizing length scale that characterizes the spread of the Burgers vector. The stress field due to the Burgers vector distribution $\tilde{w}(\mathbf{x} ; a)$ is then expressed as the convolution of $\tilde{w}(\mathbf{x} ; a)$ with the classical Volterra solution $\sigma_{\alpha \beta}(\mathbf{x})$ as

$$
\tilde{\sigma}_{\alpha \beta}(\mathbf{x} ; a)=\int \sigma_{\alpha \beta}\left(\mathbf{x}-\mathbf{x}^{\prime}\right) \tilde{w}\left(\mathbf{x}^{\prime} ; a\right) \mathrm{d}^{3} \mathbf{x}^{\prime}
$$

In DDD simulations, the chief quantity of interest is not the local stress but the interaction force between different dislocation segments. In non-singular theory, this involves the interaction between the two spread cores and thus, a double convolution. The associated stress field $\sigma_{\alpha \beta}^{\text {ns }}$ that yields the correct Peach-Koehler force, $\mathrm{d} \mathbf{F}=$

$\boldsymbol{\sigma}^{\mathrm{ns}} \mathbf{b} \wedge \mathrm{d} \mathbf{L}$ on an elementary segment $\mathrm{d} \mathbf{L}$ of a dislocation of Burgers vector $\mathbf{b}$, is called the non-singular field, and is given as [1]

$$
\sigma_{\alpha \beta}^{\mathrm{ns}}(\mathbf{x})=\int \sigma_{\alpha \beta}\left(\mathbf{x}-\mathbf{x}^{\prime}\right) w\left(\mathbf{x}^{\prime} ; a\right) \mathrm{d}^{3} \mathbf{x}^{\prime}
$$


where $w(\mathbf{x} ; a)$ is the convolution of $\tilde{w}(\mathbf{x} ; a)$ with itself, i.e.

$$
w(\mathbf{x} ; a)=\int \tilde{w}\left(\mathbf{x}-\mathbf{x}^{\prime} ; a\right) \tilde{w}\left(\mathbf{x}^{\prime} ; a\right) \mathrm{d}^{3} \mathbf{x}^{\prime}
$$

An analytical expression for $w(\mathbf{x})$ was derived as [1]

$$
w(\mathbf{x} ; a)=\frac{15}{8 \pi a^{3}\left(r^{2} / a^{2}+1\right)^{7 / 2}}
$$

and analytic results for the non-singular stress fields of edge, screw and linear combinations of any dislocation character could also be obtained.

The elastic energy of a dislocation is computed, with $S_{i j k l}$ the compliance tensor, as

$$
E=\frac{1}{2} \int S_{i j k l} \tilde{\sigma}_{i j} \tilde{\sigma}_{k l} \mathrm{~d} v
$$

This requires the stress field $\tilde{\sigma}_{\alpha \beta}$ rather than the non-singular field, $\sigma_{\alpha \beta}^{\text {ns }}$. An exact analytic form for $\tilde{w}(\mathbf{x} ; a)$ and thus $\tilde{\sigma}_{\alpha \beta}$ is not available. However, it was found [1] that a very good analytic approximation to $\tilde{w}(\mathbf{x} ; a)$ is

$$
\tilde{w}(\mathbf{x} ; a) \approx(1-m) w\left(\mathbf{x} ; a_{1}\right)+m w\left(\mathbf{x} ; a_{2}\right)
$$

with $a_{1}=0.9038 a, a_{2}=0.5451 a, m=0.6575$. Therefore, using (14) in (9) and noting the definition in $(10), \tilde{\sigma}_{\alpha \beta}(\mathbf{x})$ is approximated as

$$
\tilde{\sigma}_{\alpha \beta}(\mathbf{x} ; a) \approx(1-m) \sigma_{\alpha \beta}^{\mathrm{ns}}\left(\mathbf{x} ; a_{1}\right)+m \sigma_{\alpha \beta}^{\mathrm{ns}}\left(\mathbf{x} ; a_{2}\right)
$$

With the analytical solutions for $\sigma_{\alpha \beta}^{\text {ns }}$ of the infinite straight non-singular dislocations in hand, the approximation for $\tilde{\sigma}_{\alpha \beta}(\mathbf{x})$ can be computed. For the screw dislocation, the non-singular stress field is

$$
\sigma_{\theta z}^{\mathrm{ns}}=\frac{\mu b}{2 \pi} \frac{r}{\rho_{a}^{2}}\left(1+\frac{a^{2}}{\rho_{a}^{2}}\right)
$$

where $\rho_{a}=\sqrt{x^{2}+y^{2}+a^{2}}$. Employing the relation (15), $\tilde{\sigma}_{\theta z}$ can be computed and the non-singular energy per unit length follows as

$$
E\left(\theta=0^{\circ}\right)=\int_{0}^{R} \frac{\left(\tilde{\sigma}_{\theta z}\right)^{2}}{2 \mu} 2 \pi r \mathrm{~d} r=\frac{\mu b^{2}}{4 \pi}\left[\ln \left(\frac{R}{a}\right)+0.49445\right]
$$

The final result is given for the proper limiting case $R \gg a$. For the edge dislocation, the total energy is calculated using a similar procedure as

$$
\begin{aligned}
E\left(\theta=90^{\circ}\right)= & \int_{0}^{R} r \mathrm{~d} r \int_{0}^{2 \pi} \mathrm{d} \theta\left[\frac{1}{2 \mu} \tilde{\sigma}_{x y}^{2}+\frac{1}{2 \mu(1+\nu)}\left(\tilde{\sigma}_{x x}^{2}+\tilde{\sigma}_{y y}^{2}-2 \nu \tilde{\sigma}_{x x}{\tilde{\sigma_{y y}}}^{2}-{\tilde{\sigma_{z z}}}^{2}\right)\right] \\
& =\frac{\mu b^{2}}{4 \pi(1-\nu)}\left[\ln \left(\frac{R}{a}\right)+\frac{0.24445-0.25 \nu-0.49445 \nu^{2}}{1-\nu^{2}}\right]
\end{aligned}
$$

The leading terms for each case coincide with the elastic strain energy obtained from the classical Volterra solution with cutoff $r_{\mathrm{c}}=a$. There is then an additional constant energy for both (17) and (18); within the general formulation of equation (2), this constant could be interpreted as an effective core energy embedded in NS theory that is associated with the regularization of the core region. 
In the singular and non-singular isotropic theories, the dislocation energy for any character angle can be decomposed into screw and edge components. So only the above results for the screw and edge dislocation are required to consider all character angles. As noted earlier, ParaDis allows for an additional core energy term proportional to the elastic energy [22] but we have seen above that this term itself does not match atomistics. Furthermore, this term was found insufficient for capturing the behavior for the bow-out of atomistic dislocations [20].

The non-singular dislocation energy follows the general form of (1). Taking the regularization parameter $a$ equal to the cutoff radius $r_{\mathrm{c}}$, the total energy of an infinite straight dislocation line in non-singular theory can be made to match the atomistic result by adding the true atomistic core energy while subtracting the additional constant energy appearing in non-singular theory. Operationally, we add a core energy correction to the non-singular energy of

$$
\begin{aligned}
E_{\mathrm{c}}^{\mathrm{corr}}(\theta)=\left.E_{\mathrm{c}}\right|_{r_{\mathrm{c}}}(\theta)-K(\theta) \ln \left(\frac{r_{\mathrm{c}}}{a}\right)-\left(\frac{0.49445 \mu b^{2}}{4 \pi}\right) \cos ^{2} \theta \\
-\left(\frac{\mu b^{2}}{4 \pi(1-\nu)} \frac{0.24445-0.25 \nu-0.49445 \nu^{2}}{1-\nu^{2}}\right) \sin ^{2} \theta
\end{aligned}
$$

with $\left.E_{\mathrm{c}}\right|_{r_{\mathrm{c}}}(\theta)$ expressed with $\left.E_{\mathrm{c}}\right|_{b}(\theta)$ as in (2), where $\left.E_{\mathrm{c}}\right|_{b}(\theta)$ is represented using (3).

With this correction, the total energy of the non-singular theory matches the true atomistic energy. We introduce the above correction by revising the definition of the core energy function in ParaDiS using the functional form for the atomistic core energy shown in figure 3 . In the actual implementation, we modified the self force due to the core energy accordingly.

\section{Validation of atomistically-informed DDD: dislocation bow-out}

We now examine a canonical dislocation problem, periodic dislocation bow-out, to assess the accuracy of DDD relative to full atomistic simulations for curved dislocations. We use the non-singular theory as implemented within the ParaDiS code [3] but augmented with the atomistically-informed core energy via the correction of (19).

The geometry of the bow-out problem is shown schematically in figure 5 . In the atomistic simulations, a single dislocation is pinned periodically by fixing a small group of atoms at the zero-stress core structure. The system is then subjected to an applied shear stress, which causes the dislocation to bow-out in between the pinning points. The simulations were reported previously [20], and were performed in a multiscale simulation that eliminates all spurious image forces that can arise in finite-size periodic simulations [27, 28]; key details are as follows. Creation of a dislocation in the atomistic region of the multiscale simulation is achieved by displacing all atoms according to the Volterra solution and then relaxing the entire multiscale system under zero displacements on the outer boundary of the system. Loading is then applied by imposing displacement boundary conditions on the outer surface of the continuum region to create the desired homogeneous pure shear stress acting on the glide plane in the direction of glide. The 
simulations are performed using explicit dynamics at $\mathrm{T}=0 \mathrm{~K}$. The pinning obstacles in the bow-out problem are created by fixing all atoms within a small cubic volume 1 $\mathrm{nm}^{3}$ centered on the dislocation core and with periodicity $L$. The interactions of the dislocation with these rigid obstacles has been estimated to be equivalent to an applied shear stress of less than $3 \mathrm{MPa}[20]$, which is small relative to the loads applied here. The full shape of the bowed-out dislocation is measured at each applied load, for both initial screw and edge dislocations. The center line of the dislocation is then determined as the mid-point between the centers of the two partial dislocations identified using the common neighbor analysis. The corresponding DDD problem is also shown in figure 5. The fixed atoms of the atomistic simulation are replaced by fixed segments of dislocation. The remaining dislocation line can bow-out in response to the applied load.

The bow-out geometry seems to be a clean geometry for measuring significant changes in dislocation curvature, and thus testing the accuracy of DDD across a range of dislocation character angles. The line tension also generally scales linearly with the maximum bow-out, enabling differences in bow-out to be interpreted in terms of approximate differences in line tension. A similar bow-out geometry was recently used [19] to test the measurement of the core line tension at finite temperature from the thermal fluctuations of straight dislocations. The simulation were, however, carried out only in the range of small bow-out amplitudes induced by thermal fluctuations.
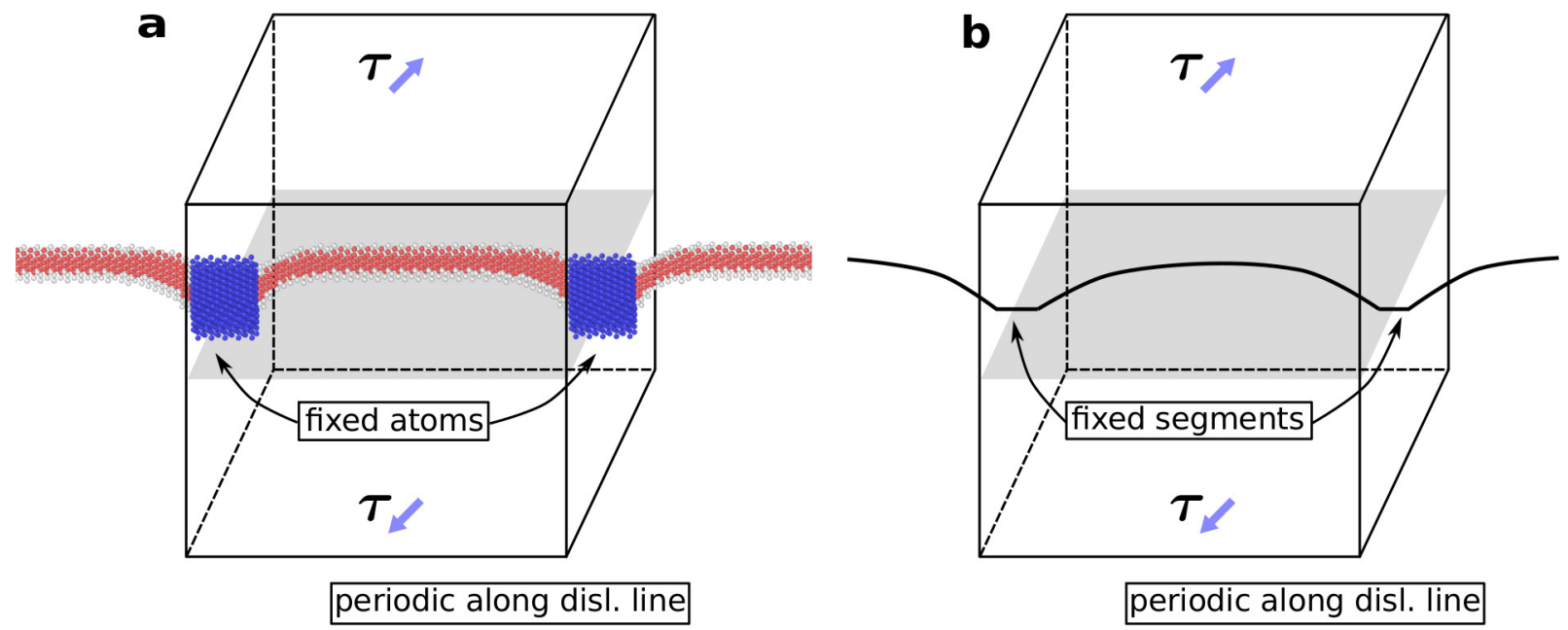

Figure 5. Atomistic bow-out simulation (a) is carried out with a multiscale scheme [27], while the DDD simulations (b) are performed in a large simulation box with periodic boundary condition along the dislocation line. The dislocation bows out in the slip plane rendered in gray in the figures. In the schematic atomistic simulation the atoms are colored by common neighbor analysis showing the stacking fault, partial dislocations and the fixed atoms; note that the schematic is not to scale.

Current DDD codes based on analytical expressions of dislocation interactions are mainly limited to elastically isotropic materials. Cubic metals are generally elastically anisotropic, and so direct comparisons between atomistic simulations and DDD are best performed for nearly-isotropic atomistic models. For this reason, our 
comparison is restricted to Aluminum modeled with two EAM potentials, [17] and [16]. For both potentials the Zener anisotropy $A=2 C_{44} /\left(C_{11}-C_{12}\right)$ values are $\mathrm{A}=1.31$, 1.21 respectively. Al is thus fairly isotropic and the effects of anisotropy may be modest. Future studies using a new anisotropic version of ParaDiS or DDD frameworks based on eigenstrains [29, 30] can avoid this small difference and extend the study to strongly anisotropic systems such as $\mathrm{Ni}$ and $\mathrm{Cu}$. Here, for DDD, we must select appropriate isotropic elastic constants for the weakly-anisotropic Al. We employ the Scattergood and Bacon effective material moduli [31] defined as $\mu_{\mathrm{s}}=4 \pi K\left(0^{\circ}\right) / b^{2}$ and $\nu_{\mathrm{s}}=1-K\left(0^{\circ}\right) / K\left(90^{\circ}\right)$. This estimate has been investigated recently [32] and shown to reproduce well the line tension of straight dislocations with different characters.

The non-singular DDD method also involves the regularization parameter $a$. For consistency of the energies in DDD with those of the atomistic simulations, this parameter must be taken equal to the cutoff parameter $r_{\mathrm{c}}$ used in the calibration of the atomistic core energy, which sets the partition between the elastic and core contributions to the dislocation energy. We use the atomistic core energy determined at $r_{c}=b$ and then, for any choice of $a$, we set $r_{c}=a$ and use the relation in (2) to get the corresponding core energy at $r_{c}=a$, then we correct it using (19) for NS theory (notice that $r_{c}=a$ gives no $K(\theta) \ln \left(r_{\mathrm{c}} / a\right)$ term). For a straight dislocation, this partitioning is artificial; the total energy is preserved for any choice of $r_{c}=a$. However, for curved dislocations, the choice of $a$ may affect the short-range interactions between dislocation segments and so may therefore affect the equilibrium dislocation geometry. In addition, non-asymptotic terms (e.g. higher order terms as $\frac{a^{2}}{R^{2}}$ ) in the total energy may enter into the DDD problem. In the following subsection, we thus probe these features numerically by investigating the effect of different regularization parameters $a$ on the predicted dislocation bow-out. Setting $a=r_{\mathrm{c}}$ in ParaDiS and introducing the atomistic core energy as in (19), we predict the bow-out in DDD for four values of $a(a=b, e b, 2 e b, 4 e b$, where $e=2.713$ is the natural logarithm base) to include the value $a=e b$ considered in earlier work [20].

In most cases, deviations between atomistic and ParaDiS DDD simulations are due to a sharper angle predicted by DDD near the pinning points. In these regions, the dislocation shape is influenced by the periodic interactions with nearby segments, which may be influenced by dislocation partial dissociation distance and the DDD treatment of the dislocation as a perfect dislocation. Also, fixing the atomic positions in the pinning regions of the atomistic simulations disrupts locally the elastic behavior of the medium and may affect the way the dislocation segments interact on either side of the obstacles. These details cannot be resolved, and we thus focus attention more on the overall extent of bow-out.

Results for the Ercolessi-Adams potential are shown in figures 6. Overall, the DDD simulations are in good agreement with the atomistic results. The differences in bowout distance at the peak - which are approximately proportional to the line tension are typically within $5 \AA$ across all cases. Furthermore, the influence of $a$ is, overall, fairly small especially at small $a$. This indicates that the calibration to the atomistic core energy captures the dominant energetic effects for curved dislocations over a range 
of curvatures. The agreement for $a=2 e b$ is perhaps slightly better across all cases relative to other choices. In particular, at the highest load of $73.9 \mathrm{MPa}$ for the screw dislocation, the DDD configuration is unstable for $a=b$ but is stable and in very good agreement with atomistics for $a=2 e b$. Using an even larger value of $a=4 e b$ leads to less bow-out, i.e. higher line tension, and some underprediction of the bow-out at higher loads. The value $a=2 e b$ thus provides the best match for the Ercolessi-Adams $\mathrm{Al}$ potential. While not directly relevant to the value of $a$, the dissociation distances of the current potential are $3.5 b$ and $7.0 b$ for screw and edge bow-out respectively. The best regularization parameter $a=2 e b$ lies in this range.

For the Mishin potential, similar trends are found as shown in figure 7 . There is a deviation for the screw dislocation at stresses only slightly larger than the Peierls stress of the straight screw dislocation; this arises because the operative Peierls stress, based on loading and unloading simulations and discussed in detail by Szajewski et al. [20], is a strong function of the curvature at low curvature (low stress) while the DDD predictions use the operative Peierls stress that is appropriate for higher curvatures. There is also a systematic deviation in the bow-out for the $50 \mathrm{~nm}$ edge case, largely independent of a. The Mishin Al potential has the most unusual core contributions to the line tension (see figure 3), which may contribute somehow to the larger deviations as compared to the EA potential. Otherwise, the dependence of the bow-out on a remains modest, while the value $a=4 e b$ provides the best overall agreement with simulations relative to other choices studied, with notably better predictions for the screw dislocation across all loads. Using this potential, the dissociation distances are $3.5 b$ and $6.3 b$ for screw and edge bow-out case. The optimal parameter $a=4 e b$ does not lie in this range, but the results with $a=2 e b$ also give relative good results and lie within the range of dissociation.

Overall, the regularization parameter $a$ has a fairly small influence on the dislocation bow-out. This is important because the atomistic simulations and calibration of NS theory do not depend on the value of $a$, and so $a$ cannot be determined. The insensitivity of our results to $a$ thus implies that reasonable values of $a$ can be used with limited concern for the creation of significant error. However, as seen here for the bow-out problem, the applied stress at the point of an instability, which may be interpreted as a strength value for some process, may be affected by the choice of $a$. For both $\mathrm{Al}$ potentials studied here, based on our comparisons with the atomistic simulations, we recommend values of $a=2-4 e b$.

Analyzing dislocation fluctuations for the Mishin potential at $T=300 \mathrm{~K}$, Geslin et al [19] found optimal values of $a=1.2 b$ and $a=2 b$ for screw and edge dislocations, respectively. Since DDD implementations require a single value of $a$, independent of the dislocation character, a global optimal value of $a=1.25 b$ was found to reproduce both the long-wavelength fluctuations and the dislocation bow-out at small applied stresses, at $T=300 \mathrm{~K}$. These optimal values of $a$ are smaller than found here. However, the fluctuation method involves rather small amplitudes of atomistic bow-out and the finite temperature study likely reduces the relevant Peierls stress. These aspects make 
direct comparisons between our study and that of Geslin et al. difficult. The relatively weak dependence of our results on the value of $a$, especially at small bowouts typical of those used by Geslin et al. suggests that any differences arising between the atomistic calibrations of Geslin et al. and the present work may not have significant quantitative influence on the accurate study of dislocation behaviors at the continuum level.
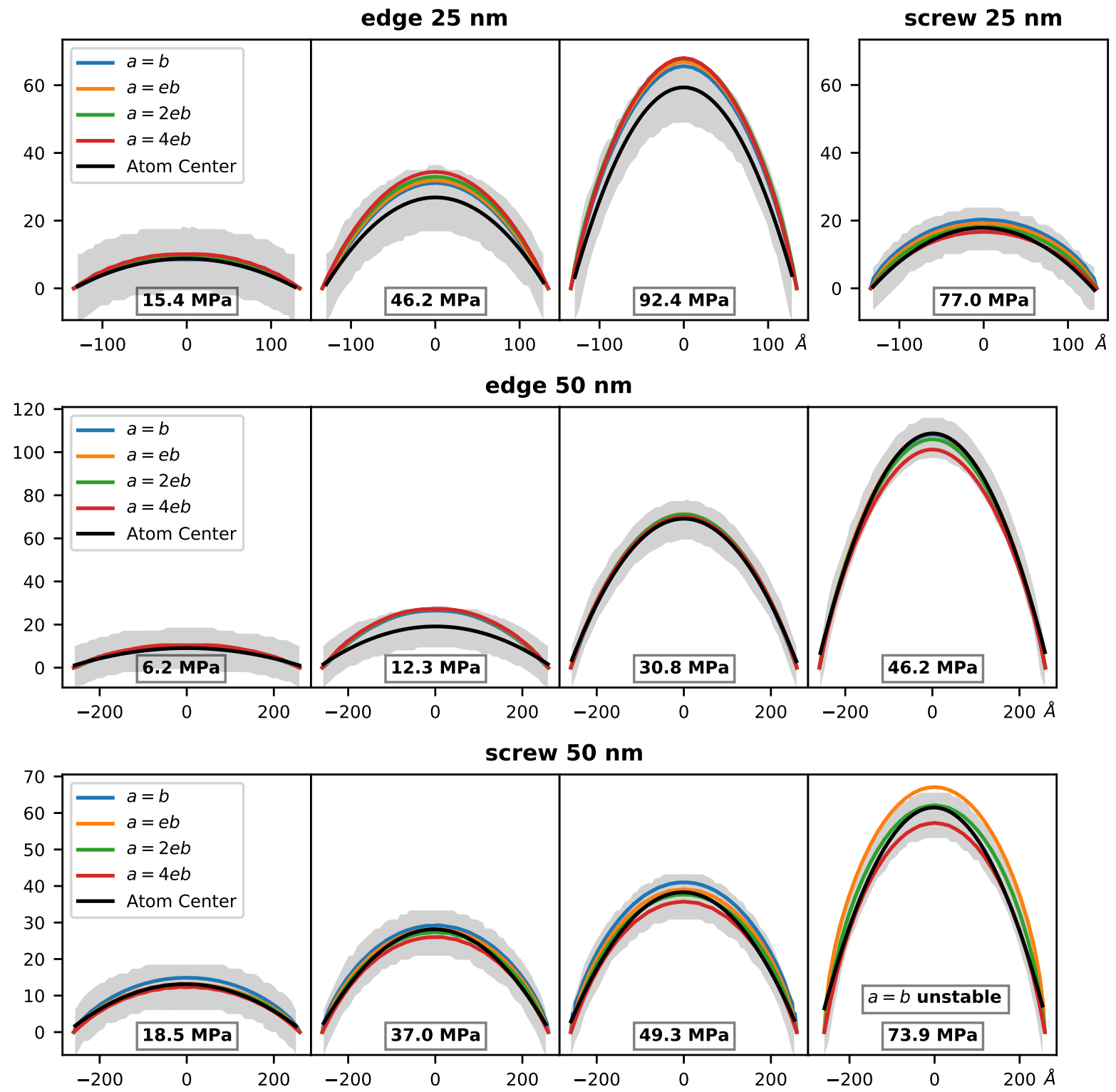

Figure 6. Comparison of atomistic simulations and ParaDiS simulations for the Ercolessi-Adams Al potential [17] using different regularization parameters a noted on the figure. The grey areas show the stacking faults. Simulations use the effective elastic moduli according to Scattergood [31]. An optimal $a=2 e b$ is found for the bow-out problem using this potential. 

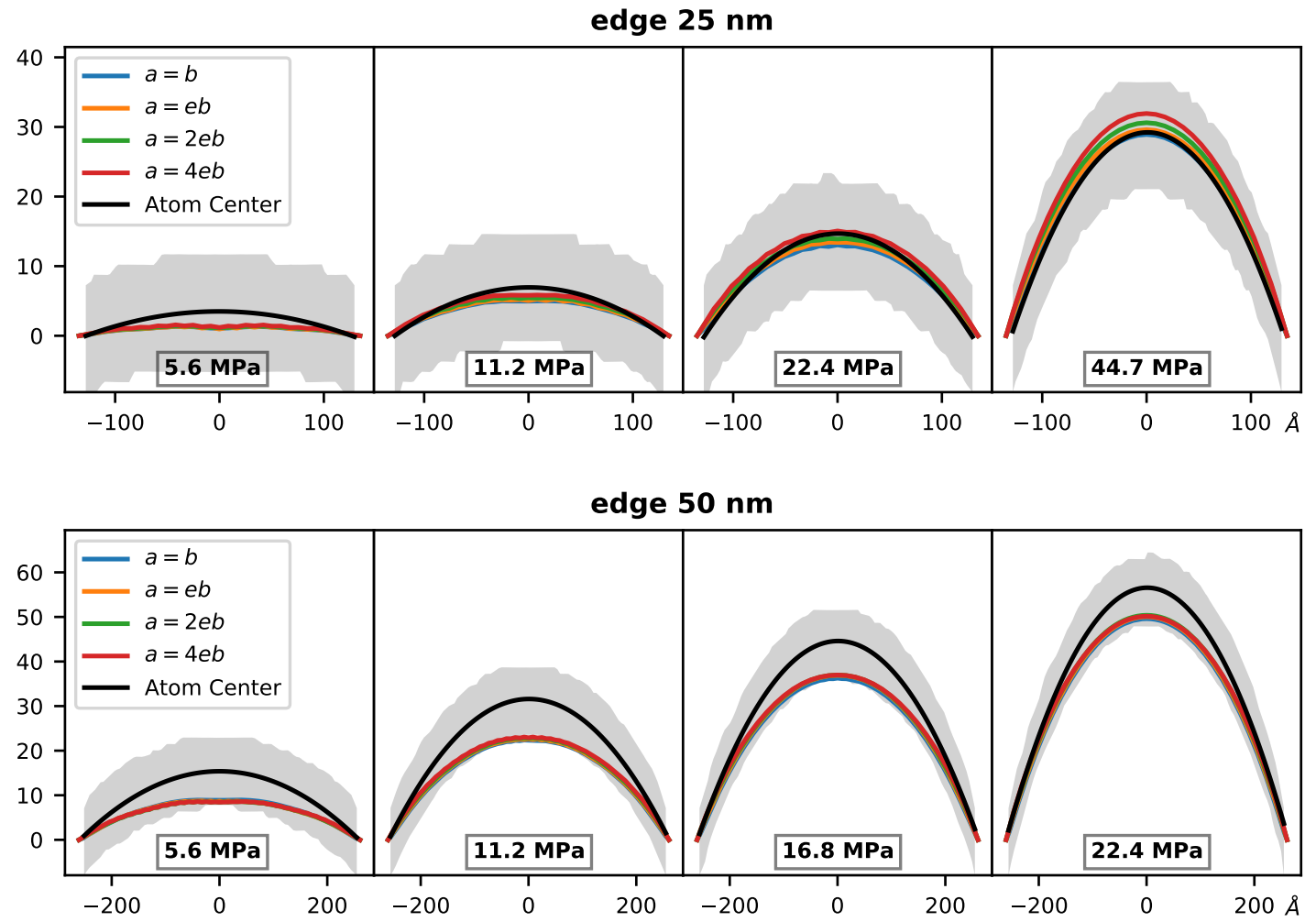

screw 25 nm

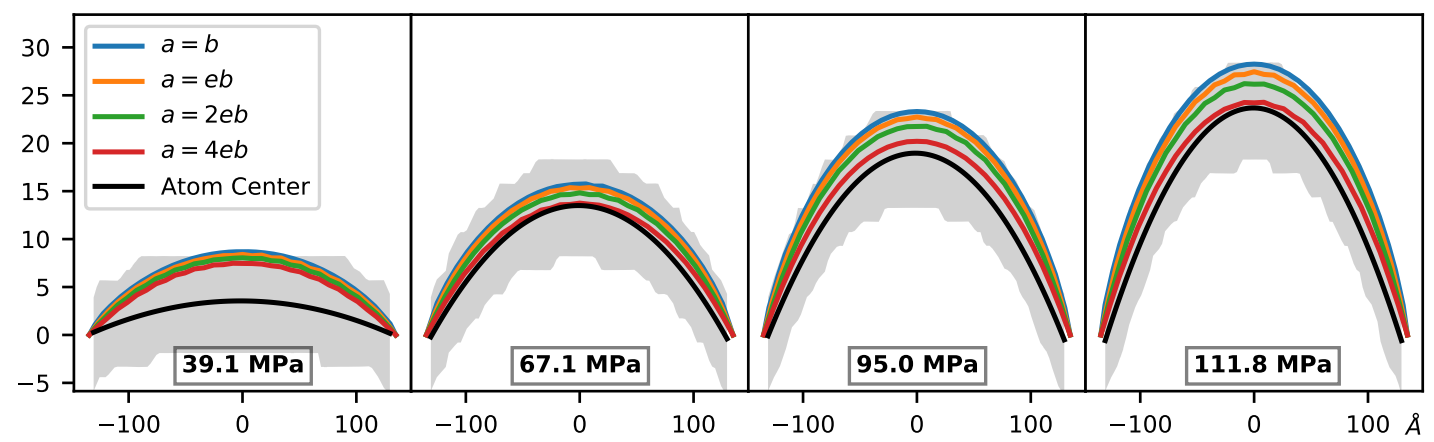

screw $50 \mathrm{~nm}$

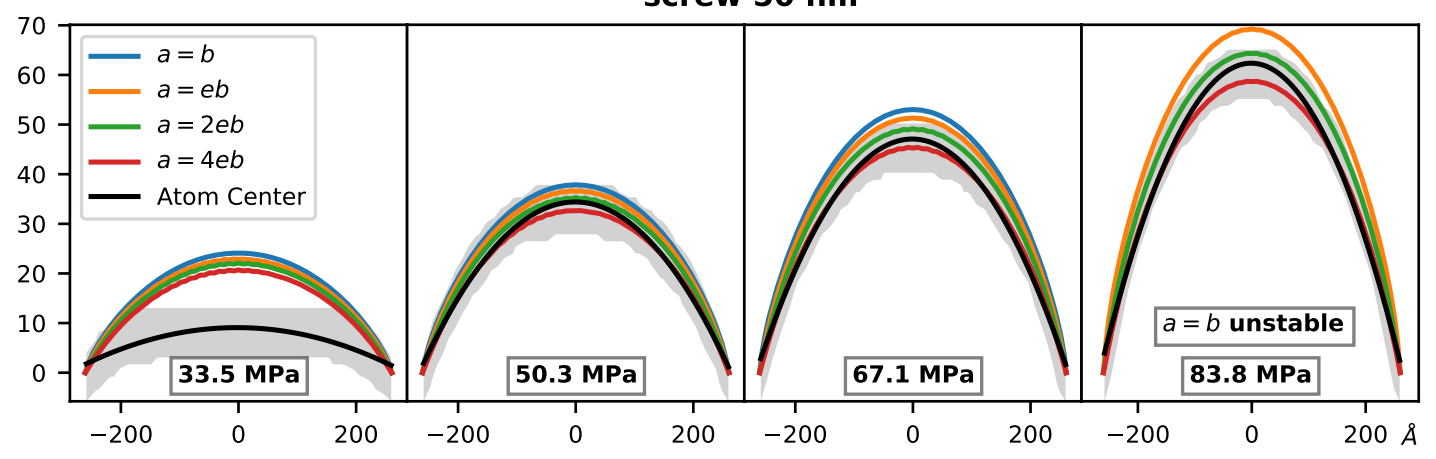

Figure 7. Comparison of atomistic simulations and ParaDiS simulations for the Mishin Al potential [16] using different regularization parameters $a$ noted on the figure. The grey areas show the stacking fault. Simulations use an effective effective moduli [31]. An optimal $a=4 e b$ is found for the bow-out problem using this potential. The lower loading cases are strongly influenced by the Peierls stress discussed in [20]. Applying an operative Peierls stress does not give good agreement with atomistics. 
The results for bow-out here differ substantially from those found previously using the standard ParaDiS core energy implementation as a fitting parameter[20]. Specifically, Szajewski et al. found that fitting of the core energy to the simulations for atomistic edge bow-out then led to very poor predictions of the bow-out for screw dislocations. The details embedded in the atomistic core energy are thus necessary if DDD studies are to be adequately representative of atomistic results.

\section{Calibration of partial dislocation core energy with NS theory}

In fcc metals, a full dislocation dissociates into two partial dislocations with a stacking fault in between. So far in this paper, we have considered the energetics of the dissociation process as part of the core energy of the full dislocation. The reason is that DDD studies rarely include the dissociation phenomenon due to the high computational cost of resolving the nanoscale partial separation. However, there are scenarios [33] where it is important to simulate the behavior of individual partial dislocations or the dissociated full dislocation within a DDD framework [10]. Therefore, in this section we compute the atomistic core energy for partial dislocations as a function of partial dislocation character in the context of NS theory.

From (17) and (18), we write the elastic energy in NS theory for any character angle $\theta$ as

$$
E^{\mathrm{ns}}(\theta)=K^{\mathrm{ns}}(\theta) \ln \left(\frac{R}{a}\right)+C(\theta)
$$

with the energy factor $K^{\mathrm{ns}}(\theta)$ and additional term $C(\theta)$ expressed as

$$
K^{\mathrm{ns}}(\theta)=\frac{\mu b^{2}}{4 \pi}\left[\cos ^{2} \theta+\frac{\sin ^{2} \theta}{(1-\nu)}\right], \quad C(\theta)=\frac{\mu b^{2}}{4 \pi}\left[C_{\mathrm{s}} \cos ^{2} \theta+\frac{C_{\mathrm{e}} \sin ^{2} \theta}{(1-\nu)}\right]
$$

where $C_{\mathrm{s}}$ and $C_{\mathrm{e}}$ are the constants in (17) and (18).

To derive the partial core energy, we need to calculate the interaction energy of the two partials within NS theory. Following [34], the interaction energy for partials separated by $r_{12}$ is the work done by the displacement of one partial with partial Burgers vector $\mathbf{b}_{2 \mathrm{p}}$ moving against the stress field $\boldsymbol{\sigma}_{1 \mathrm{p}}^{\mathrm{ns}}$ of the second partial from large separation $R$ to separation $r_{12}$. We decompose the partial Burgers vector into screw and edge component as $\mathbf{b}_{2 \mathrm{p}}=\mathbf{b}_{2 \mathrm{ps}}+\mathbf{b}_{2 \mathrm{pe}}$. From isotropic elasticity, the stress field can be written as $\boldsymbol{\sigma}_{1 \mathrm{p}}^{\mathrm{ns}}=\boldsymbol{\sigma}_{1 \mathrm{ps}}^{\mathrm{ns}}+\boldsymbol{\sigma}_{1 \mathrm{pe}}^{\mathrm{ns}}$ representing the field associated with the screw and edge components of the Burgers vector. We denote the dislocation line direction as $\boldsymbol{\xi}$ and employ the stress field of a straight dislocation in [1] to obtain the Peach-Koehler force on the other dislocation. Then the interaction energy can be derived as

$$
\begin{aligned}
W_{12}^{\mathrm{ns}} & =\int_{r_{12}}^{R}\left(\left(\boldsymbol{\sigma}_{1 \mathrm{ps}}^{\mathrm{ns}}+\boldsymbol{\sigma}_{1 \mathrm{pe}}^{\mathrm{ns}}\right) \cdot\left(\mathbf{b}_{2 \mathrm{ps}}+\mathbf{b}_{2 \mathrm{pe}}\right)\right) \times \boldsymbol{\xi} \cdot \mathrm{d} \mathbf{s} \\
& =\frac{\mu b_{1 \mathrm{ps}} b_{2 \mathrm{ps}}}{4 \pi}\left[\ln \left(\frac{R^{2}+a^{2}}{r_{12}^{2}+a^{2}}\right)+a^{2}\left(\frac{1}{r_{12}^{2}+a^{2}}-\frac{1}{R^{2}+a^{2}}\right)\right]+\frac{\mu b_{1 \mathrm{pe}} b_{2 \mathrm{pe}}}{4 \pi(1-\nu)} \ln \left(\frac{R^{2}+a^{2}}{r_{12}^{2}+a^{2}}\right)
\end{aligned}
$$


We note that in equation (22) the partial Burgers vectors correspond to $\left|\mathbf{b}_{1 \mathrm{p}}\right|=\left|\mathbf{b}_{2 \mathrm{p}}\right|=$ $|\mathbf{b}| / \sqrt{3}$. Combining the logarithm terms in (22), we rewrite the interaction energy as

$$
W_{12}^{\mathrm{ns}}=K_{12}^{\mathrm{ns}} \ln \left(\frac{R^{2}+a^{2}}{r_{12}^{2}+a^{2}}\right)+C_{\mathrm{p} 12}
$$

with the $K_{12}^{\mathrm{ns}}$ as the prelogarithmic factor and $C_{\mathrm{p} 12}$ as the constants due to NS theory.

The total dislocation energy consists of the interaction energy, the stacking fault energy over length $r_{12}$, and the partial core energies $E_{\text {cp }}$. In NS theory, the total energy is thus

$$
\begin{aligned}
E= & E_{\mathrm{p} 1}+E_{\mathrm{p} 2}+W_{12}+\gamma_{\mathrm{sf}} r_{12} \\
= & \left(K_{\mathrm{p} 1}^{\mathrm{ns}} \ln \left(\frac{R}{a}\right)+C_{\mathrm{p} 1}+E_{\mathrm{cp} 1}^{\mathrm{ns}}\right)+\left(K_{\mathrm{p} 2}^{\mathrm{ns}} \ln \left(\frac{R}{a}\right)+C_{\mathrm{p} 2}+E_{\mathrm{cp} 2}^{\mathrm{ns}}\right) \\
& +\left(K_{12}^{\mathrm{ns}} \ln \left(\frac{R^{2}}{r_{12}^{2}+a^{2}}\right)+C_{\mathrm{p} 12}\right)+\gamma_{\mathrm{sf}} r_{12} \\
= & K^{\mathrm{ns}} \ln \left(\frac{R}{a}\right)+\left(K_{12}^{\mathrm{ns}} \ln \left(\frac{a^{2}}{r_{12}^{2}+a^{2}}\right)+\gamma_{\mathrm{sf}} r_{12}+C_{\mathrm{p} 1}+C_{\mathrm{p} 2}+C_{\mathrm{p} 12}\right)+E_{\mathrm{cp} 1}^{\mathrm{ns}}+E_{\mathrm{cp} 2}^{\mathrm{ns}}
\end{aligned}
$$

where (20) and (23) are used, and we take the appropriate limit of large $R \gg a$.

Calculation of the partial core energy in (24) requires knowledge of the equilibrium partial dissociation distance $r_{12}$. The equilibrium dissociation distance in NS theory minimizes the total dislocation energy, i.e. satisfies $\frac{\partial E}{\partial r_{12}}=0$, leading to

$$
-\frac{2 K_{12}^{\mathrm{ns}} r_{12}}{r_{12}^{2}+a^{2}}-\frac{\mu b_{1 \mathrm{~s}} b_{2 \mathrm{~s}}}{2 \pi} \frac{a^{2} r_{12}}{\left(r_{12}^{2}+a^{2}\right)^{2}}+\gamma_{\mathrm{sf}}=0
$$

The NS value for $r_{12}$ is always smaller than the value $K_{12} / \gamma_{\mathrm{sf}}$ derived from singular theory (obtained by setting $a=0$ in NS theory). More importantly, for Al, both the NS and singular theory estimates are not in good agreement with atomistic simulations, as shown in figure 8 . Better agreement is obtained for materials with lower stable stacking fault energies, such as $\mathrm{Cu}$ and $\mathrm{Ni}$, with correspondingly larger $r_{12}$ values. Additionally, for these more anisotropic materials, an anisotropic elastic analysis needs to be carried out. However, the difference between atomistic and NS predictions for $r_{12}$ immediately implies that the derived partial core energies are only effective core energies that include some contributions associated with the stacking fault energy and elastic interactions. However, within the closed system of a DDD simulation using the NS description of partial dislocations, the use of the NS value for $r_{12}$ is the only self-consistent approach that will also accurately capture the total dislocation energy.

Using the NS dissociation distance and the dissociation geometry shown by the Thompson tetrahedron (figure 9), we reformulate equation (24) as

$$
E(\theta)=K^{\mathrm{ns}}(\theta) \ln \left(\frac{R}{a}\right)+M(\theta)+E_{\mathrm{cp}}^{\mathrm{ns}}\left(\theta-30^{\circ}\right)+E_{\mathrm{cp}}^{\mathrm{ns}}\left(\theta+30^{\circ}\right)
$$



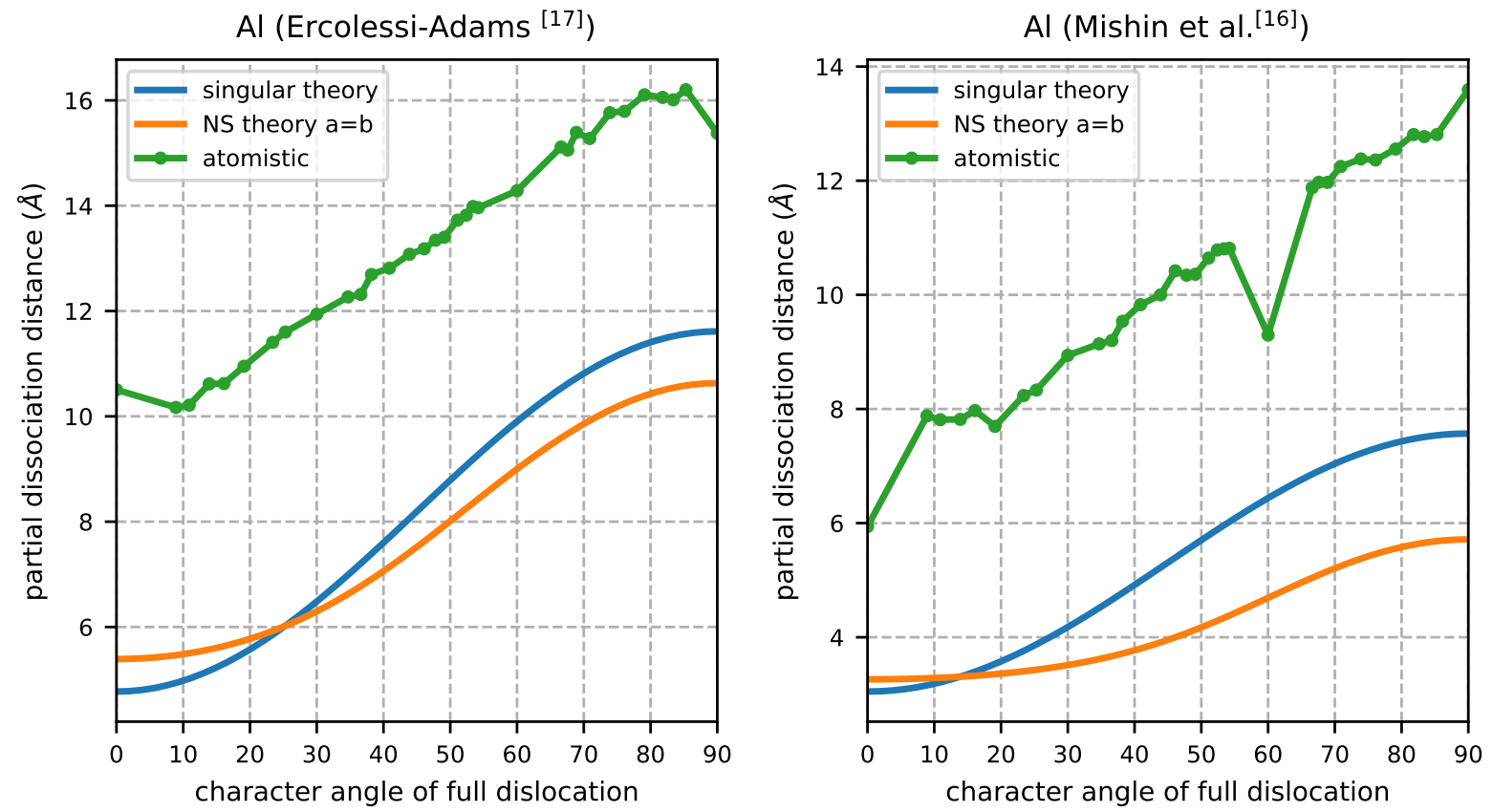

Figure 8. Comparison between the dissociation distance measured in atomistic simulations and predicted from (25) with the singular $(a=0)$ and non-singular $(a=b)$ theories.

with the notation

$$
\begin{aligned}
& M(\theta)=K_{12}^{\mathrm{ns}}(\theta) \ln \left(\frac{a^{2}}{r_{12}^{2}+a^{2}}\right)+\gamma_{\mathrm{sf}} r_{12}(\theta)+C_{\mathrm{p} 1}(\theta)+C_{\mathrm{p} 2}(\theta)+C_{\mathrm{p} 12}(\theta) \\
& C_{\mathrm{p} 1}(\theta)=\frac{\mu b^{2}}{12 \pi}\left(C_{\mathrm{s}} \cos ^{2}\left(\theta-30^{\circ}\right)+\frac{C_{\mathrm{e}}}{1-\nu} \sin ^{2}\left(\theta-30^{\circ}\right)\right) \\
& C_{\mathrm{p} 2}(\theta)=\frac{\mu b^{2}}{12 \pi}\left(C_{\mathrm{s}} \cos ^{2}\left(\theta+30^{\circ}\right)+\frac{C_{\mathrm{e}}}{1-\nu} \sin ^{2}\left(\theta+30^{\circ}\right)\right) \\
& C_{\mathrm{p} 12}(\theta)=\frac{\mu b^{2}}{12 \pi}\left(\frac{a^{2}}{r_{12}^{2}+a^{2}} \cos \left(\theta-30^{\circ}\right) \cos \left(\theta+30^{\circ}\right)\right) \\
& C_{\mathrm{s}}=0.49445, \quad C_{\mathrm{e}}=\frac{0.24445-0.25 \nu-0.49445 \nu^{2}}{1-\nu^{2}}
\end{aligned}
$$

Subtracting the overall elastic energy contribution that is independent of the partials and dissociation, the total dislocation core energy is

$$
E_{\mathrm{ct}}(\theta)=M(\theta)+E_{\mathrm{cp}}^{\mathrm{ns}}\left(\theta-30^{\circ}\right)+E_{\mathrm{cp}}^{\mathrm{ns}}\left(\theta+30^{\circ}\right)
$$



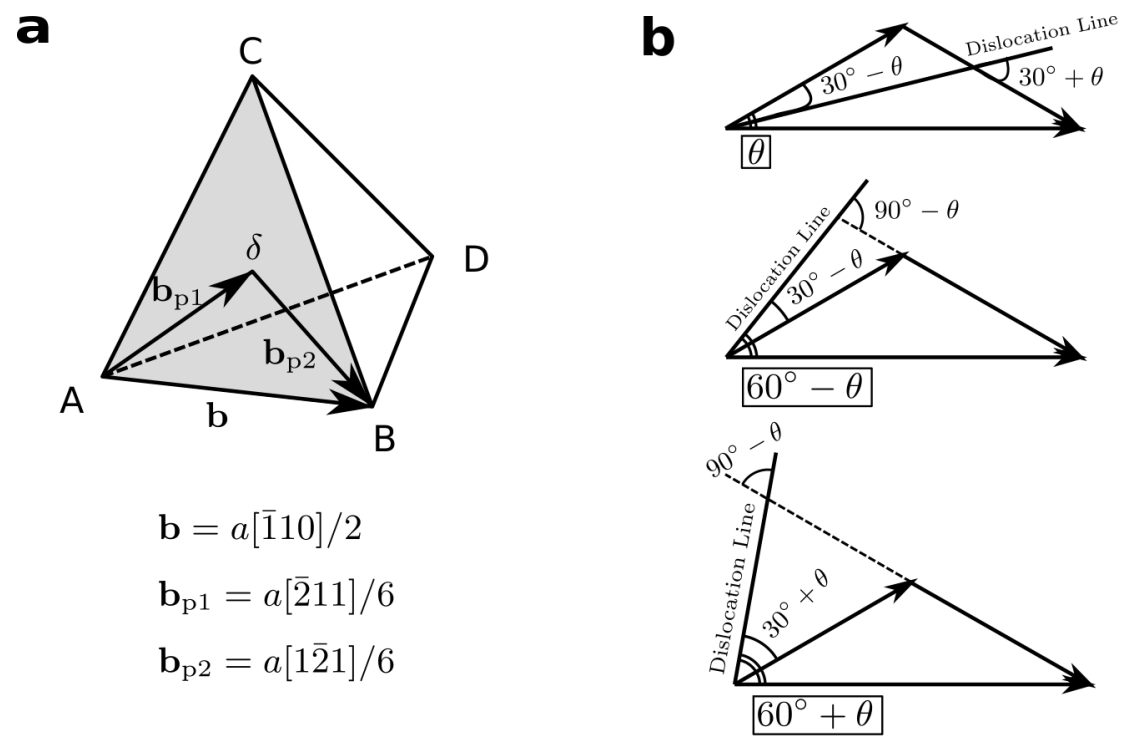

Figure 9. (a) Thompson tetrahedron ABCD. A perfect dislocation with Burgers vector $\mathbf{b}$ dissociates into two partial dislocations having Burgers vectors $\mathbf{b}_{\mathrm{p} 1}$ and $\mathbf{b}_{\mathrm{p} 2}$ on the same slip plane. (b) Three different perfect dislocation orientations $\theta,\left(60^{\circ}-\theta\right)$ and $\left(60^{\circ}+\theta\right)$ are used to extract the partial core energies.

To solve for the partial core energy $E_{\mathrm{cp}}^{\mathrm{ns}}(\theta)$ on the left hand side of (28), we examine the dislocation dissociation geometry and search for common partial dislocation orientations. For a dislocation with character angle $\theta$, the associated partial dislocations have character angles $\left(30^{\circ}-\theta\right)$ and $\left(30^{\circ}+\theta\right)$. The full dislocation orientations on the right of figure 9 lead to the following set of equations

$$
\begin{aligned}
& E_{\mathrm{cp}}^{\mathrm{ns}}\left(30^{\circ}-\theta\right)+E_{\mathrm{c}}^{\mathrm{ns}}\left(30^{\circ}+\theta\right)=E_{\mathrm{ct}}(\theta)-M(\theta) \\
& E_{\mathrm{cp}}^{\mathrm{ns}}\left(30^{\circ}+\theta\right)+E_{\mathrm{cp}}^{\mathrm{ns}}\left(90^{\circ}-\theta\right)=E_{\mathrm{ct}}\left(60^{\circ}+\theta\right)-M\left(60^{\circ}+\theta\right) \\
& E_{\mathrm{cp}}^{\mathrm{ns}}\left(30^{\circ}-\theta\right)+E_{\mathrm{cp}}^{\mathrm{ns}}\left(90^{\circ}-\theta\right)=E_{\mathrm{ct}}\left(60^{\circ}-\theta\right)-M\left(60^{\circ}-\theta\right)
\end{aligned}
$$

Summing the last two equations in (29) and subtracting the first equation, we obtain the partial core energy at character angle $\left(90^{\circ}-\theta\right)$ as

$$
\begin{aligned}
E_{\mathrm{cp}}^{\mathrm{ns}}\left(90^{\circ}-\theta\right)= & \frac{1}{2}\left[E_{\mathrm{ct}}\left(60^{\circ}-\theta\right)+E_{\mathrm{ct}}\left(60^{\circ}+\theta\right)-E_{\mathrm{ct}}(\theta)\right. \\
& \left.-M\left(60^{\circ}-\theta\right)-M\left(60^{\circ}+\theta\right)+M(\theta)\right]
\end{aligned}
$$

Substituting $\left(90^{\circ}-\theta\right)$ with $\theta$, we rewrite the above equation as

$$
\begin{aligned}
E_{\mathrm{cp}}^{\mathrm{ns}}(\theta)= & \frac{1}{2}\left[E_{\mathrm{ct}}\left(\theta-30^{\circ}\right)+E_{\mathrm{ct}}\left(\theta+30^{\circ}\right)-E_{\mathrm{ct}}\left(90^{\circ}-\theta\right)\right. \\
& \left.-M\left(\theta-30^{\circ}\right)-M\left(\theta+30^{\circ}\right)+M\left(90^{\circ}-\theta\right)\right]
\end{aligned}
$$

In the above derivation, the symmetry and periodicity properties of the dislocation core energy are used, i.e. $E_{\mathrm{c}}(\theta)=E_{\mathrm{c}}(-\theta), E_{\mathrm{c}}(\theta)=E_{\mathrm{c}}\left(180^{\circ}-\theta\right)$ for both partial and full core energies. Hence with the full core energy approximation in figure 3 , the elastic interaction energy factor $K_{12}^{\text {ns }}$ from (22), the solved dissociation estimation $r_{12}$ in (25) and the stable stacking fault energy $\gamma_{\mathrm{sf}}$, the NS partial core energy as a function of partial character is computed for the two $\mathrm{Al}$ potentials as shown in figure 10. 
For comparison, we also compute the partial core energy using the singular theory (Volterra dislocation, $a=0$ ). The NS correction additional $C_{\mathrm{p} 1}, C_{\mathrm{p} 2}, C_{\mathrm{p} 12}$ vanish and we re-introduce the cutoff radius $r_{\mathrm{c}}$ as in equation (1). Using the dissociation distance from singular theory, we obtain the partial core energy versus character for the two $\mathrm{Al}$ potentials as shown in figure 10 .

The two approaches yield surprisingly similar core energies for pure edge partials but increasingly different values as the character changes toward pure screw. Neither analysis is preferable over the other - they differ due to the different self-consistent treatments of the dislocation (non-singular and singular) - and both are ultimately calibrated to the correct total energy of the full dislocation.
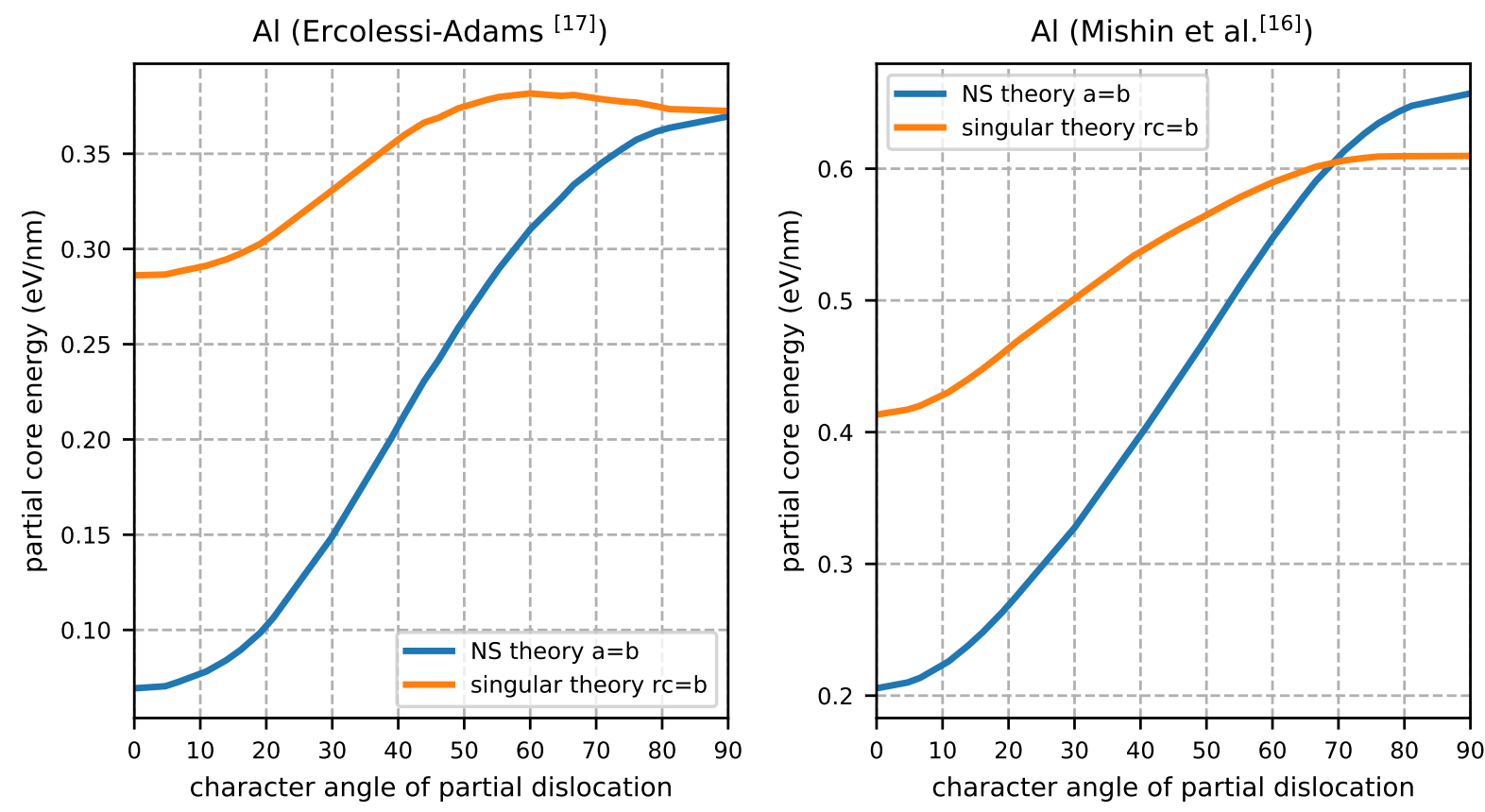

Figure 10. Partial core energy with two Al potentials calibrated from both the singular and non-singular theories. The approximations for the full core energy in figure 3 were used.

The inclusion of partial dislocations into DDD simulations may have some implications for dislocation network evolution. Some years ago, the study of dislocation junction structures and their destruction under load [35] showed some agreement between atomistic and DDD predictions. However, the critical resolved shear stresses for junction destruction differed, which may be due to the absence of an atomisticallycalibrated core energy in the DDD method. Other work embedding atomistic information into DDD only used the atomistic stacking fault energy investigated in [10] and inclusion of an accurate core energy may further improve the quantitative agreement between atomistic simulations and DDD. However, for anisotropic materials, the full comparison requires the use of an anisotropic DDD code. 


\section{Discussion and Summary}

Atomistic simulations have been used to compute the dislocation core energy at cutoff scale $r_{c}=b$ as a function of character angle for $\mathrm{Al}, \mathrm{Cu}$, and $\mathrm{Ni}$ as described by well-established interatomic potentials. The core energy at any other cut-off can be determined analytically, with all choices preserving the total (elastic + core) dislocation energy. The proper incorporation of the atomistic core energy into the non-singular theory of dislocations for any value of the NS regularization parameter $a$ has been presented and implemented in the DDD code ParaDiS. Comparisons of the calibrated DDD predictions against full atomistic studies have been shown for the problem of dislocation bow-out. Over a range of lengths, loads, and for edge and screw dislocations, the calibrated DDD model for nearly-isotropic $\mathrm{Al}$ is in good agreement with simulations. Only modest variations in the DDD bow-out are found as a function of the NS regularization parameter $a$, although the values $a=2 e b \sim 4 e b$ give the best agreement with atomistic simulations for $\mathrm{Al}$ using two different interatomic potentials. We further demonstrated how to compute the core energies for partial dislocations in fcc metals based on the total energies computed atomistically and the stable stacking fault energy.

The present method of calibration does not, in principle, involve any approximations. The accuracy of the core energy quantity can be controlled and reduced while the calibration of NS theory is precise within the context of NS theory. Comparisons of atomistics and DDD so far do involve the assumption that the weak anisotropy of $\mathrm{Al}$ is not quantitatively important, and results show that there is some residual dependence of the DDD results on $a$ that is outside the scope of the calibration. However, these issues are not unique to the present method of determining the core energy itself, but rather to the more general issues of the implementations of DDD. The calibration of partial core energies does involve the use of the NS partial separation, which differs from the atomistic value, but this is consistent for implementations of DDD using NS. Core corrections for NS theory of partial dislocation interactions must also be included. However, the methodology for computing partial core energies from simulations of full dislocations is generally valid, and our results here could be applied to DDD models using singular descriptions [5, 4] or improved non-singular models [9, 39].

The present calibration method using a single dislocation in a large cylindrical geometry is very straightforward to execute for any elastically anisotropic system and for any dislocation character and type (glissile, sessile). The method also enables

the accuracy and size-convergence of the core energy to be easily determined. The competing and commonly-used geometry of periodic arrays of dislocations provides a perfectly fine alternative to the present method, and has been widely used. The dipole method does require, in principle, some study of convergence of the total energy versus size. It also requires the numerical calculation of anisotropic elastic corrections for the image dislocations [40], which necessitates implementation of additional codes. To our knowledge, the corrections to NS theory for periodic dipole configurations have not been determined analytically, adding a bit of complexity to application of the dipole method. 
As noted earlier, however, the periodic dipole method is necessary for estimating core energies from first-principles simulations because this geometry does not involve any surfaces on the outer boundaries that can lead to additional contributions to the total energy that make extraction of the core energy inaccurate.

The careful calibration of the core energy to a particular atomistic potential as present here drives simulation studies toward more-quantitative results. Core energy contributions to DDD influence the predicted critical stresses for dislocations to cut or loop precipitates and to break dislocation junctions, and so will enable better quantification between DDD and atomistic studies. In addition, careful calibration now enables more-reliable execution of coupled multiscale methods that have atomistic resolution in critical small domains with DDD resolution in much larger domains [36, 37, 38], where high consistency between atomistic and DDD representations of dislocations is essential. Finally, the accurate study of the core energies of different interatomic potentials of the same metal provides an understanding of one source of uncertainty in atomistic predictions of dislocation phenomena relative to the real metal.

\section{Acknowledgments}

YH and WAC acknowledge support for this work by the NCCR MARVEL, funded by the Swiss National Science Foundation.

\section{References}

[1] Cai W, Arsenlis A, Weinberger C R and Bulatov V V 2006 A non-singular continuum theory of dislocations Journal of the Mechanics and Physics of Solids 54 561-587

[2] Hirth J P and Lothe J 1982 Theory of Dislocations (Krieger Publishing Company)

[3] Arsenlis A, Cai W, Tang M, Rhee M, Oppelstrup T, Hommes G, Pierce T G and Bulatov V V 2007 Enabling strain hardening simulations with dislocation dynamics Modelling and Simulation in Materials Science and Engineering $\mathbf{1 5} 553$

[4] Po G, Mohamed M S, Crosby T, Erel C, El-Azab A and Ghoniem N 2014 Recent Progress in Discrete Dislocation Dynamics and Its Applications to Micro Plasticity The Journal of The Minerals, Metals \& Materials Society 66 2108-2120

[5] Weygand D, Friedman L H, Giessen E V d and Needleman A 2002 Aspects of boundary-value problem solutions with three-dimensional dislocation dynamics Modelling and Simulation in Materials Science and Engineering $10437-468$

[6] Devincre B and Condat M 1992 Model validation of a 3D simulation of dislocation dynamics: Discretization and line tension effects Acta Metallurgica et Materialia 40 2629-2637

[7] Fivel M C 2008 Discrete dislocation dynamics: an important recent break-through in the modelling of dislocation collective behaviour Comptes Rendus Physique 9 427-436

[8] Krebs J, Rao S I, Verheyden S, Miko C, Goodall R, Curtin W A and Mortensen A 2017 Cast aluminium single crystals cross the threshold from bulk to size-dependent stochastic plasticity Nature Materials 16 730-736

[9] Lazar M 2013 The fundamentals of non-singular dislocations in the theory of gradient elasticity: Dislocation loops and straight dislocations International Journal of Solids and Structures $\mathbf{5 0}$ $352-362$

[10] Martínez E, Marian J, Arsenlis A, Victoria M and Perlado J M 2008 Atomistically informed dislocation dynamics in fcc crystals Journal of the Mechanics and Physics of Solids 56 869-895 
[11] Curtin W A and Miller R E 2003 Atomistic/continuum coupling in computational materials science Modelling and Simulation in Materials Science and Engineering 11 R33-R68

[12] Xiong L, Deng Q, Tucker G, McDowell D L and Chen Y 2012 A concurrent scheme for passing dislocations from atomistic to continuum domains Acta Materialia 60 899-913

[13] Anderson P M, Hirth J P and Lothe J 2017 Theory of Dislocations 3rd ed (Cambridge: Cambridge University Press)

[14] Zhou X W, Sills R B, Ward D K and Karnesky R A 2017 Atomistic calculations of dislocation core energy in aluminium Physical Review B 95054112

[15] Li J, Wang C Z, Chang J P, Cai W, Bulatov V V, Ho K M and Yip S 2004 Core energy and Peierls stress of a screw dislocation in bcc molybdenum: A periodic-cell tight-binding study Physical Review B $\mathbf{7 0} 104113$

[16] Mishin Y, Farkas D, Mehl M J and Papaconstantopoulos D A 1999 Interatomic potentials for monoatomic metals from experimental data and ab initio calculations Physical Review B $\mathbf{5 9}$ 3393-3407

[17] Ercolessi F and Adams J B 1994 Interatomic potentials from first-principles calculations: the force-matching method EPL (Europhysics Letters) $\mathbf{2 6} 583$

[18] Geslin P A, Gatti R, Devincre B and Rodney D 2017 Implementation of the nudged elastic band method in a dislocation dynamics formalism: Application to dislocation nucleation Journal of the Mechanics and Physics of Solids 108 49-67

[19] Geslin P A and Rodney D 2018 Thermal fluctuations of dislocations reveal the interplay between their core energy and long-range elasticity Physical Review B 98174115

[20] Szajewski B A, Pavia F and Curtin W A 2015 Robust atomistic calculation of dislocation line tension Modelling and Simulation in Materials Science and Engineering 23085008

[21] Stroh A N 1958 Dislocations and cracks in anisotropic elasticity Philosophical Magazine 3 625-646

[22] Fitzgerald S P and Aubry S 2010 Self-force on dislocation segments in anisotropic crystals Journal of Physics: Condensed Matter 22295403

[23] Hirth J P, Jøssang T and Lothe J 1966 Dislocation energies and the concept of line tension Journal of Applied Physics 37 110-116

[24] Lardner R W 1971 Mathematical Theory of Dislocations and Fracture (University of Toronto Press)

[25] Lothe J 1992 Chapter 2 - Dislocations in Continuous Elastic Media Elastic Strain Fields and Dislocation Mobility (Modern Problems in Condensed Matter Sciences vol 31) (Elsevier) 175235

[26] Varvenne C, Leyson G P M, Ghazisaeidi M and Curtin W A 2017 Solute strengthening in random alloys Acta Materialia 124 660-683

[27] Pavia F and Curtin W A 2015 Parallel algorithm for multiscale atomistic/continuum simulations using LAMMPS Modelling and Simulation in Materials Science and Engineering 23055002

[28] Szajewski B A and Curtin W A 2015 Analysis of spurious image forces in atomistic simulations of dislocations Modelling and Simulation in Materials Science and Engineering 23025008

[29] Jamond O, Gatti R, Roos A and Devincre B 2016 Consistent formulation for the DiscreteContinuous Model: Improving complex dislocation dynamics simulations International Journal of Plasticity 80 19-37

[30] Bertin N and Capolungo L 2018 A FFT-based formulation for discrete dislocation dynamics in heterogeneous media Journal of Computational Physics 355 366-384

[31] Scattergood R O and Bacon D J 1975 The Orowan mechanism in anisotropic crystals Philosophical Magazine 31 179-198

[32] Szajewski B A, Hunter A, Luscher D J and Beyerlein I J 2018 The influence of anisotropy on the core structure of Shockley partial dislocations within FCC materials Modelling and Simulation in Materials Science and Engineering 26015010

[33] Rodney D and Phillips R 1999 Structure and strength of dislocation junctions: an atomic level analysis Physical Review Letters 82 1704-1707

[34] Wu Z, Yin B and Curtin W A 2016 Energetics of dislocation transformations in hcp metals Acta 
Materialia 119 203-217

[35] Madec R, Devincre B, Kubin L, Hoc T and Rodney D 2003 The role of collinear interaction in dislocation-induced hardening Science 301 1879-1882

[36] Anciaux G, Junge T, Hodapp M, Cho J, Molinari J F and Curtin W A 2018 The Coupled Atomistic/Discrete-Dislocation method in 3d part I: Concept and algorithms Journal of the Mechanics and Physics of Solids 118 152-171

[37] Hodapp M, Anciaux G, Molinari J F and Curtin W A 2018 Coupled atomistic/discrete dislocation method in 3D Part II: Validation of the method Journal of the Mechanics and Physics of Solids $1191-19$

[38] Cho J, Molinari J F, Curtin W A and Anciaux G 2018 The coupled atomistic/discrete-dislocation method in 3d. Part III: Dynamics of hybrid dislocations Journal of the Mechanics and Physics of Solids 118 1-14

[39] Boleininger M, Swinburne T D, and Dudarev S L 2018 Atomistic-to-continuum description of edge dislocation core: Unification of the Peierls-Nabarro model with linear elasticity Physical Review Materials 2083803

[40] Cai W, Bulatov V V, Chang J P, Li J, and Yip S 2003 Periodic image effects in dislocation modelling Philosophical Magazine 83 539-567

[41] Clouet E, Ventelon E, and Willaime F 2009 Dislocation Core Energies and Core Fields from First Principles Physical Review Letters 102055502

[42] Gurrutxaga-Lerma B and Verschueren J 2018 Elastic models of dislocations based on atomistic Kanzaki forces Physical Review B 98134104

[43] Po G, Lazar M, Admal N C and Ghoniem N 2018 International Journal of Plasticity 103 1-22 